\title{
New ultracool subdwarfs identified in large-scale surveys using Virtual Observatory tools ${ }^{\star, \star \star}$
}

\section{UKIDSS LAS DR5 vs. SDSS DR7}

\author{
N. Lodieu ${ }^{1,2}$, M. Espinoza Contreras ${ }^{1}$, M. R. Zapatero Osorio ${ }^{3}$, E. Solano ${ }^{4,5}$, M. Aberasturi ${ }^{4,5}$, and E. L. Martín ${ }^{3}$ \\ 1 Instituto de Astrofísica de Canarias (IAC), Calle Vía Láctea s/n, 38200 La Laguna, Tenerife, Spain \\ e-mail: [nlodieu;marcela]@iac.es \\ 2 Departamento de Astrofísica, Universidad de La Laguna (ULL), 38205 La Laguna, Tenerife, Spain \\ 3 Centro de Astrobiología (CSIC-INTA), Ctra. Ajalvir km 4, 28850 Torrejón de Ardoz, Madrid, Spain \\ e-mail: [mosorio; ege]@cab.inta-csic.es \\ ${ }^{4}$ Centro de Astrobiología (INTA-CSIC), Departamento de Astrofísica, PO Box 78, 28691 Villanueva de la Cañada, Madrid, Spain \\ e-mail: [esm;miriam]@cab.inta-csic.es \\ 5 Spanish Virtual Observatory
}

Received 22 December 2011 / Accepted 16 April 2012

\section{ABSTRACT}

\begin{abstract}
Aims. The aim of the project is to improve our knowledge of the low-mass and low-metallicity population to investigate the influence of metallicity on the stellar (and substellar) mass function.

Methods. We present the results of a photometric and proper motion search aimed at discovering ultracool subdwarfs in large-scale surveys. We employed and combined the Fifth Data Release (DR5) of the UKIRT Infrared Deep Sky Survey (UKIDSS) Large Area Survey (LAS) and the Sloan Digital Sky Survey (SDSS) Data Release 7 complemented with ancillary data from the Two Micron All-Sky Survey (2MASS), the DEep Near-Infrared Survey (DENIS) and the SuperCOSMOS Sky Surveys (SSS).

Results. The SDSS DR7 vs. UKIDSS LAS DR5 search returned a total of 32 ultracool subdwarf candidates, only two of which are recognised as a subdwarf in the literature. Twenty-seven candidates, including the two known ones, were followed-up spectroscopically in the optical between 600 and $1000 \mathrm{~nm}$, thus covering strong spectral features indicative of low metallicity (e.g., CaH), 21 with the Very Large Telescope, one with the Nordic Optical Telescope, and five were extracted from the Sloan spectroscopic database to assess (or refute) their low-metal content. We confirm 20 candidates as subdwarfs, extreme subdwarfs, or ultra-subdwarfs with spectral types later than M5; this represents a success rate of $\geq 60 \%$. Among those 20 new subdwarfs, we identify two early-L subdwarfs that are very likely located within $100 \mathrm{pc}$, which we propose as templates for future searches because they are the first examples of their subclass. Another seven sources are solar-metallicity M dwarfs with spectral types between M4 and M7 without H $\alpha$ emission, suggesting that they are old M dwarfs. The remaining five candidates do not have spectroscopic follow-up yet; only one remains as a bona-fide ultracool subdwarf after revision of their proper motions. We assigned spectral types based on the current classification schemes and, when possible, we measured their radial velocities. Using the limited number of subdwarfs with trigonometric parallaxes, we estimated distances ranging from $\sim 95$ to $\sim 600 \mathrm{pc}$ for the new subdwarfs. We provide mid-infrared photometry extracted from the WISE satellite databases for two subdwarfs and discuss their colours. Finally, we estimate a lower limit of the surface density of ultracool subdwarfs about 5000-5700 times lower than that of solar-metallicity late-M dwarfs.
\end{abstract}

Key words. subdwarfs - techniques: photometric - techniques: spectroscopic - infrared: stars - surveys - virtual observatory tools

\section{Introduction}

Cool subdwarfs are metal-deficient population-II dwarfs that appear less luminous than their solar-metallicity counterparts due to the dearth of metals in their atmospheres (Baraffe et al. 1997). They tend to exhibit halo or thick-disk kinematics, including noticeable proper motion and high heliocentric velocities (Gizis 1997). They are very old and represent useful tracers of the Galactic chemical history (Burgasser et al. 2003). The adopted classification for $\mathrm{M}$ subdwarfs (sdM) and extreme subdwarfs (esdM) has recently been revised by Lépine et al. (2007). A new class of subdwarfs, the ultra-subdwarfs (usdM), has been

\footnotetext{
* Based on observations made with ESO Telescopes at the La Silla Paranal Observatory under programme ID 084.C-0928A.

$\star \star$ Based on observations made with the Nordic Optical Telescope, operated on the island of La Palma jointly by Denmark, Finland, Iceland, Norway, and Sweden, in the Spanish Observatorio del Roque de los Muchachos of the Instituto de Astrofísica de Canarias.
}

added to the sdM and esdM classes originally defined by Gizis (1997). The new scheme is based on a parameter, $\zeta_{\mathrm{TiO} / \mathrm{CaH}}$, which quantifies the weakening of the strength of the TiO band (in the optical) as a function of metallicity. An alternative classification based on temperature, gravity, and metallicity has been proposed by Jao et al. (2008). The range of metallicity for subdwarfs, extreme subdwarfs, and ultra-subdwarfs span approximately -0.5 and $-1.0,-1.0$ and -1.5 and below -1.5 , respectively (Gizis 1997; Woolf et al. 2009). M-type subdwarfs have typically effective temperatures below $\sim 3500-4000 \mathrm{~K}$ (depending on the metallicity Baraffe et al. 1997; Woolf et al. 2009) and should display high gravity $(\log g \sim 5.5)$ although some variations is seen among low-metallicity spectra (Jao et al. 2008).

Subdwarfs were generally identified from optical $\left(B_{J}, R\right.$, and $I$ ) proper motion catalogues on photographic plates at different epochs (Luyten 1979, 1980; Scholz et al. 2000; Lépine et al. 2003b; Lodieu et al. 2005). Several surveys have been conducted to search for subdwarfs over a wide temperature range, including 
hot (Ryan 1989), intermediate (Yong \& Lambert 2003; Digby et al. 2003), and cool components (Gizis 1997). A growing number of $\mathrm{M}$ subdwarfs have been announced over the past years, raising the number of metal-deficient dwarfs with spectral types later than M7 to about ten (Gizis 1997; Gizis \& Reid 1997; Schweitzer et al. 1999; Lépine et al. 2003a; Scholz et al. 2004b; Cushing et al. 2009). This number increased significantly after the discovery of 23 late-type subdwarfs in the Sloan Digital Sky Survey (SDSS; Adelman-McCarthy et al. 2009) spectroscopic database (Lépine \& Scholz 2008) and 15 others in the multi-epoch database of the 2MASS survey (Kirkpatrick et al. 2010). Moreover, the hydrogen-burning limit has been crossed with the discovery of the first substellar subdwarf in the 2MASS database (Burgasser et al. 2003). This discovery was quickly followed by the announcement of another L subdwarf (Burgasser 2004) and, more recently, by seven new ones (Sivarani et al. 2009; Cushing et al. 2009; Lodieu et al. 2010; Kirkpatrick et al. 2010; Schmidt et al. 2010a; Bowler et al. 2010). This number of ultracool subdwarfs remains very small, however, and is at odds with the numerous $\mathrm{L}$ and $\mathrm{T}$ dwarfs reported in the solar neighbourhood within the framework of large-scale optical and infrared surveys, including the Two Micron All Sky Survey (2MASS; e.g. Kirkpatrick et al. 2000; Burgasser et al. 2002), DEep Near Infrared Survey (DENIS; e.g. Delfosse et al. 1997, 1999; Martín et al. 1999), SDSS (e.g. Fan et al. 2000; Leggett et al. 2000; Geballe et al. 2002), the UKIRT Infrared Deep Sky Survey (UKIDSS; e.g. Lodieu et al. 2007; Pinfield et al. 2008; Burningham et al. 2010), the Canada-France-Hawaii Brown Dwarf survey (Delorme et al. 2008; Reylé et al. 2010; Albert et al. 2011), and WISE (Kirkpatrick et al. 2011; Cushing et al. 2011).

The aim of our study is to identify a complete census of metal-poor dwarfs to bridge the gap between the coolest $\mathrm{M}$ subdwarfs and the recent $\mathrm{L}$ subdwarfs identified in various surveys. In this paper we report the outcome of a photometric and proper motion searches using the UKIRT Infrared Deep Sky Survey (UKIDSS; Lawrence et al. 2007) Large Area Survey (LAS) Data Release 5 (DR5) and the Sloan Digital Sky Survey (SDSS) Data Release 7 (DR7; Abazajian et al. 2009). This catalogue search was complemented by ancillary data from 2MASS (Cutri et al. 2003; Skrutskie et al. 2006), DENIS (DENIS Consortium 2005), and SuperCOSMOS (Hambly et al. 2001c,b,a). In Sect. 2 we describe the photometric and proper motion criteria designed to identify ultracool subdwarfs in public databases using Virtual Observatory tools. In Sect. 4 we detail the spectroscopic follow-up conducted at optical wavelengths with the visual and near UV FOcal Reducer and low dispersion Spectrograph (FORS2; Appenzeller et al. 1998) mounted on the Very Large Telescope (VLT), the ALFOSC spectrograph on the Nordic Optical Telescope (NOT) and complemented by optical spectra downloaded from the SDSS spectroscopic database. In Sect. 5 we present the results of the spectroscopic analysis and determine the main properties of the newly identified ultracool subdwarfs including colours, spectral types. radial velocities, and distances. Finally, we summarise our work in Sect. 6.

\section{Sample selection}

In this section we describe the selection procedure developed to unveil new subdwarfs in the cross-correlation of the SDSS DR7 and UKIDSS LAS DR5 catalogues.

The idea of a generic photometric search for subdwarfs was triggered by the $(I-J, J-K)$ colour-colour diagram that is presented in Fig. 4 of Scholz et al. (2004b). A similar diagram

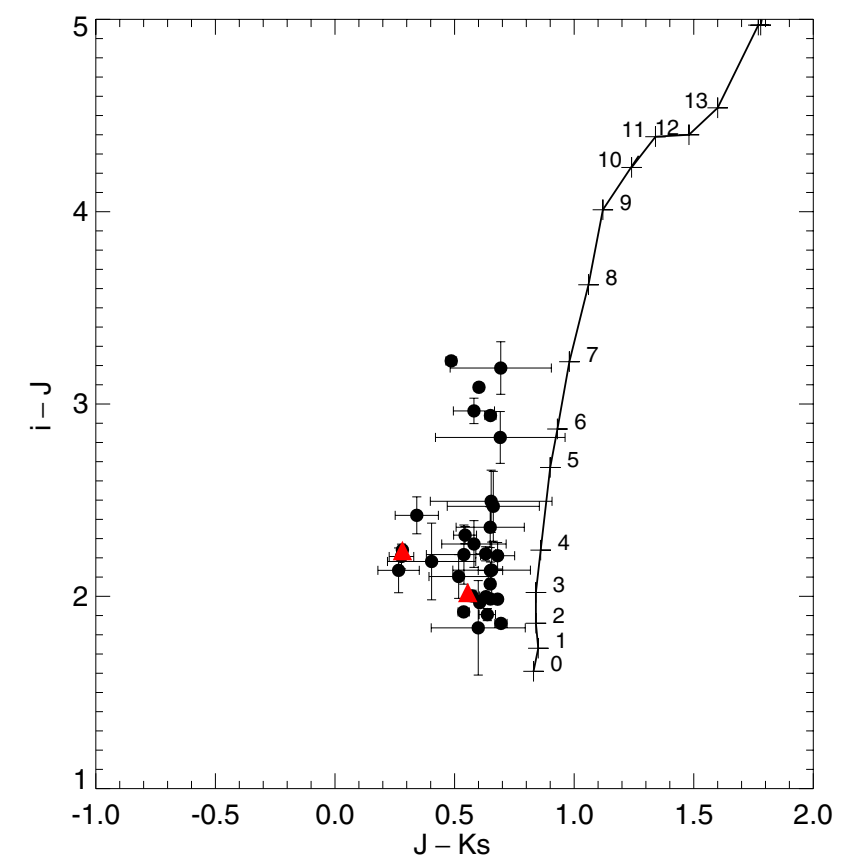

Fig. 1. $\left(J-K_{\mathrm{s}}, i-J\right)$ colour-colour diagram showing the positions of our subdwarf candidates identified in the SDSS/UKIDSS search (filled circles) with their respective error bars. Overplotted as filled red triangles are two known ultracool subdwarfs from Lépine \& Scholz (2008) with $J$ and $K$ photometry from UKIDSS LAS DR5 (error bars are smaller than the symbols) and the solar-metallicity $\mathrm{M} / \mathrm{L}$ dwarf sequence (line with crosses; spectral types are labelled; $0 \equiv \mathrm{M} 0,10 \equiv \mathrm{L} 0$ ) from West et al. (2008) and Schmidt et al. (2010b). The photometry shown in this diagram is in the MKO photometric system (Hewett et al. 2006).

is shown in Fig. 1. Known ultracool subdwarfs identified by Lépine \& Scholz (2008) in the SDSS spectroscopic database are plotted along with the sequence of field M/L dwarfs (West et al. 2008; Schmidt et al. 2010b). Subdwarfs tend to be bluer than solar metallicity $\mathrm{M} / \mathrm{L}$ dwarfs but occupy the same region in the $i-J$ domain as their solar metallicity counterparts, making their identification difficult in a pure optical-to-infrared search. However, the onset of collision-induced molecular hydrogen $\mathrm{H}_{2}$ opacity at near-infrared wavelengths generates bluer $J-H$ and $J-K_{\mathrm{s}}$ colours $\left(J-K_{\mathrm{s}} \leq 0.7\right)$ than observed for solar metallicity $\mathrm{M}$ and $\mathrm{L}$ dwarfs.

To optimise our photometric selection, we employed the reduced proper motion $\left(H_{r}=r+5 \times \log (\mu)+5\right)$ as a proxy for metallicity. This parameter is useful to separate solar-metallicity stars from subdwarfs and white dwarfs (Jones 1972; Evans 1992; Salim \& Gould 2002; Lépine \& Shara 2005; Burgasser et al. 2007; Lodieu et al. 2009). A reduced proper motion diagram is shown in Fig. 2 with known subdwarfs from Lépine \& Scholz (2008) marked as open symbols and our new candidates that are confirmed as subdwarfs shown as filled symbols.

Our search has been conducted taking advantage of Virtual Observatory (VO) ${ }^{1}$ tools, namely STILTS ${ }^{2}$ and Aladin ${ }^{3}$ (Bonnarel et al. 2000). The detailed photometric and proper motion criteria used for the SDSS DR7 vs. UKIDSS LAS DR5 search are given below. The resulting total number of candidates

\footnotetext{
1 The Virtual Observatory (http: //www . ivoa.net) is an operational research infrastructure designed to facilitate the access and analysis of the information hosted in astronomical archives.

2 http://www.star.bris.ac.uk/ mbt/stilts/

3 http://aladin.u-strasbg.fr/
} 

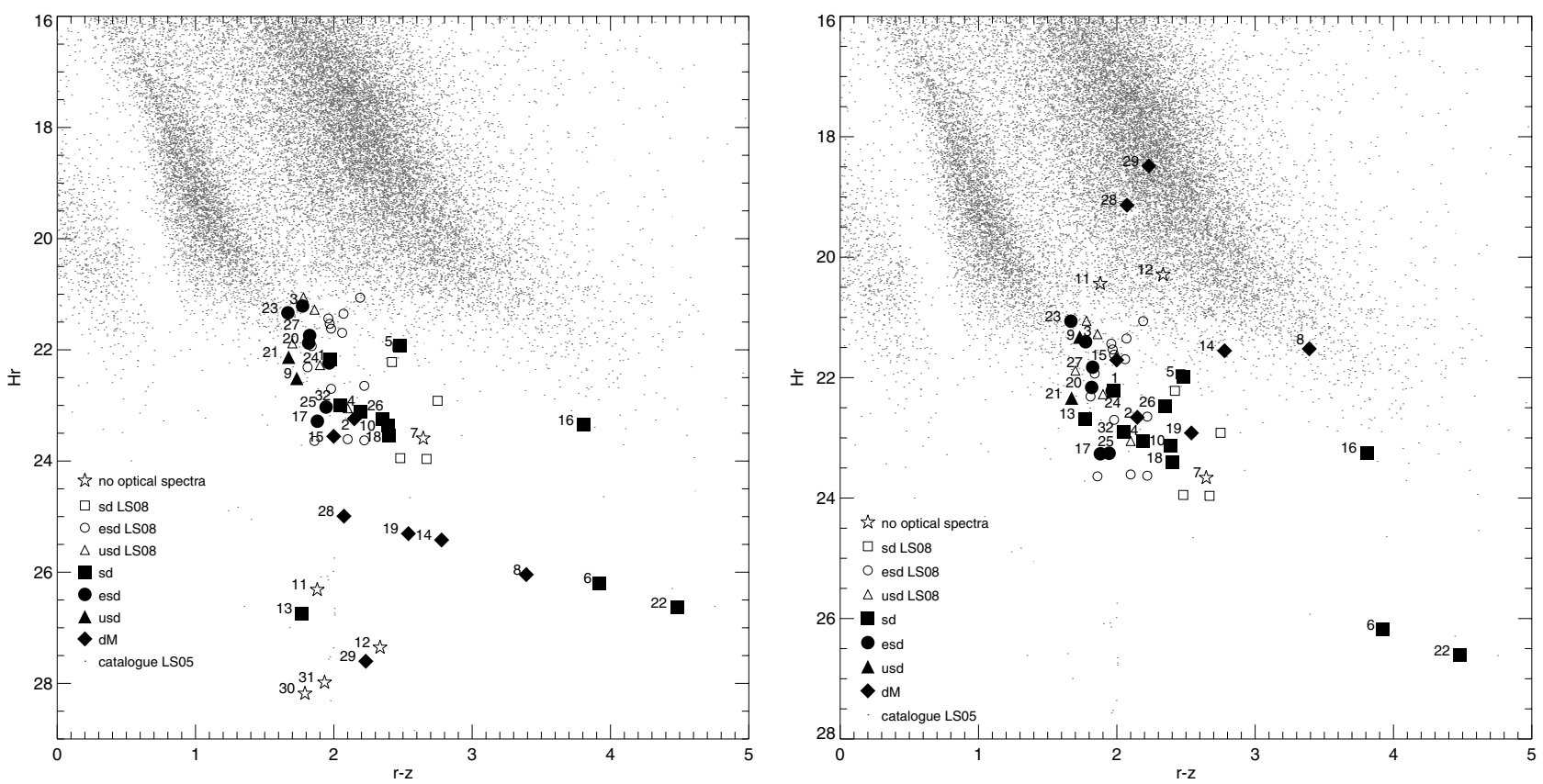

Fig. 2. Reduced proper motion diagram for new ultracool subdwarfs identified in the SDSS DR7 vs. the UKIDSS LAS DR5 search. The small dots represent all sources in the catalogue published by Lépine \& Shara (2005, LS05) with their counterpart in the SDSS DR7 database. Our new subdwarfs, extreme subdwarfs, and ultra-subdwarfs are marked as filled squares, circles, and triangles, respectively. Diamonds represent our candidates classified as solar-metallicity M dwarfs. The five sources without optical spectroscopy are marked with a star symbol. Known subdwarfs from the literature are marked as open symbols (Lépine \& Scholz 2008, LS08). Left: diagram for the values given by the Virtual Observatory. Right: diagram using the revised proper motions computed with the method detailed in Sect. 3.

returned by this query is 33 (Table 1 ) but one was rejected after looking at the images. Those candidates are shown as filled symbols in Figs. 1 and 2. The finding charts created with Aladin are displayed in Figs. A.1 and A.2 of the Appendix.

- Only SDSS point sources (class $=6$ ) were considered.

- UKIDSS point sources (mergedClass parameter equal to -1 or -2 ) and detections in $Y, J$, and $H$.

- Only sources fainter than $Y>10.5, J>10.5$, and $H>$ 10.2 mag were considered to avoid saturated stars.

- For all SDSS sources, we looked for UKIDSS counterparts between 1 and 5 arcsec, implying that we are sensitive to dwarfs with proper motions between 0.125 and $\sim 1.0$ arcsec per year depending on the baseline existing between SDSS and UKIDSS (our targets span the 3.11-7.93 year baseline).

- Colour selection was then applied as follows: $r-i \geq 1.0 \mathrm{mag}$, $g-r \geq 1.8 \mathrm{mag}, r-z \geq 1.6 \mathrm{mag}$.

- Only UKIDSS sources with good quality flags were kept i.e. ppErrBits parameter in each filter less or equal to 256.

- Infrared criterion of $J-K \leq 0.7$ mag was applied.

- Xi and Eta parameters referring to positional matching should be between -0.5 and 0.5 .

- Only objects satisfying $H_{r} \geq 20.7$ mag were kept.

The proper motion of each candidate was computed using the UKIDSS LAS and SDSS positions only, but images from 2MASS, DENIS, and SuperCOSMOS were checked by eye for additional epochs to exclude false positives. A revision of the proper motions is detailed in Sect. 3.2.

Candidates were visually inspected using the scripting capabilities of Aladin. Sources from DENIS, 2MASS, SDSS, SuperCOSMOS and UKIDSS as well images from UKIDSS, 2MASS and SDSS were used in the analysis. False candidates were rejected due to several reasons, the most frequent being the mismatch between the SDSS source and the associated UKIDSS counterpart.
We imposed a lower limit on the proper motion on purpose to bias our search towards halo objects (e.g., Scholz et al. 2000) and avoid contamination by "normal" dwarfs and extragalactic sources. Originally, we took 0.5 arcsec (instead of 1 arcsec) as the lower limit for the SDSS-UKIDSS separation, but it produced very many false candidates. Moreover, imposing a detection in the $g$-band hampers the detection of cooler subdwarfs, which would be faint at short optical wavelengths. We tested our completeness limit by checking how many of the $\sim 250$ known ultracool subdwarfs from different studies are recovered by our work (Gizis 1997; Schweitzer et al. 1999; Lépine et al. 2002, 2003b; Scholz et al. 2004b,a; Lodieu et al. 2005; Reid \& Gizis 2005; Lépine \& Scholz 2008; Kirkpatrick et al. 2010). Of the $\sim 250 \mathrm{M}$ - and L-type subdwarfs, only 11 lie in the common area between UKIDSS LAS DR5 and SDSS DR7: seven do not satisfy either optical colour constraints because they have spectral types earlier than M5 or are lacking near-infrared photometry in at least one of the three bands $(Y J H)$; two out of 11 are recovered by our search criteria: LHS 2045 (esdM4.5) and SDSS J020533.75+123824.0 (ID = 5; esdM8.5; Lépine \& Scholz 2008). However, two are not within our sample: LHS 2096 (esdM5.5; Hr $=20.62 \mathrm{mag}$ Lépine \& Shara 2005; West et al. 2008) because of the lower limit we imposed on the reduced proper motion, and SDSS J023557.61+010800.5 (esdM7; Lépine \& Scholz 2008) because of its separation between the SDSS and UKIDSS coordinates (0.92 arcsec) compared to our lower limit of 1.0 arcsec.

\section{Proper motion revision}

In this section we discuss the accuracy of the proper motions obtained by the Virtual Observatory tools by comparing the positions in the SDSS and UKIDSS catalogues (no errors considered) and dividing by the epoch difference. 


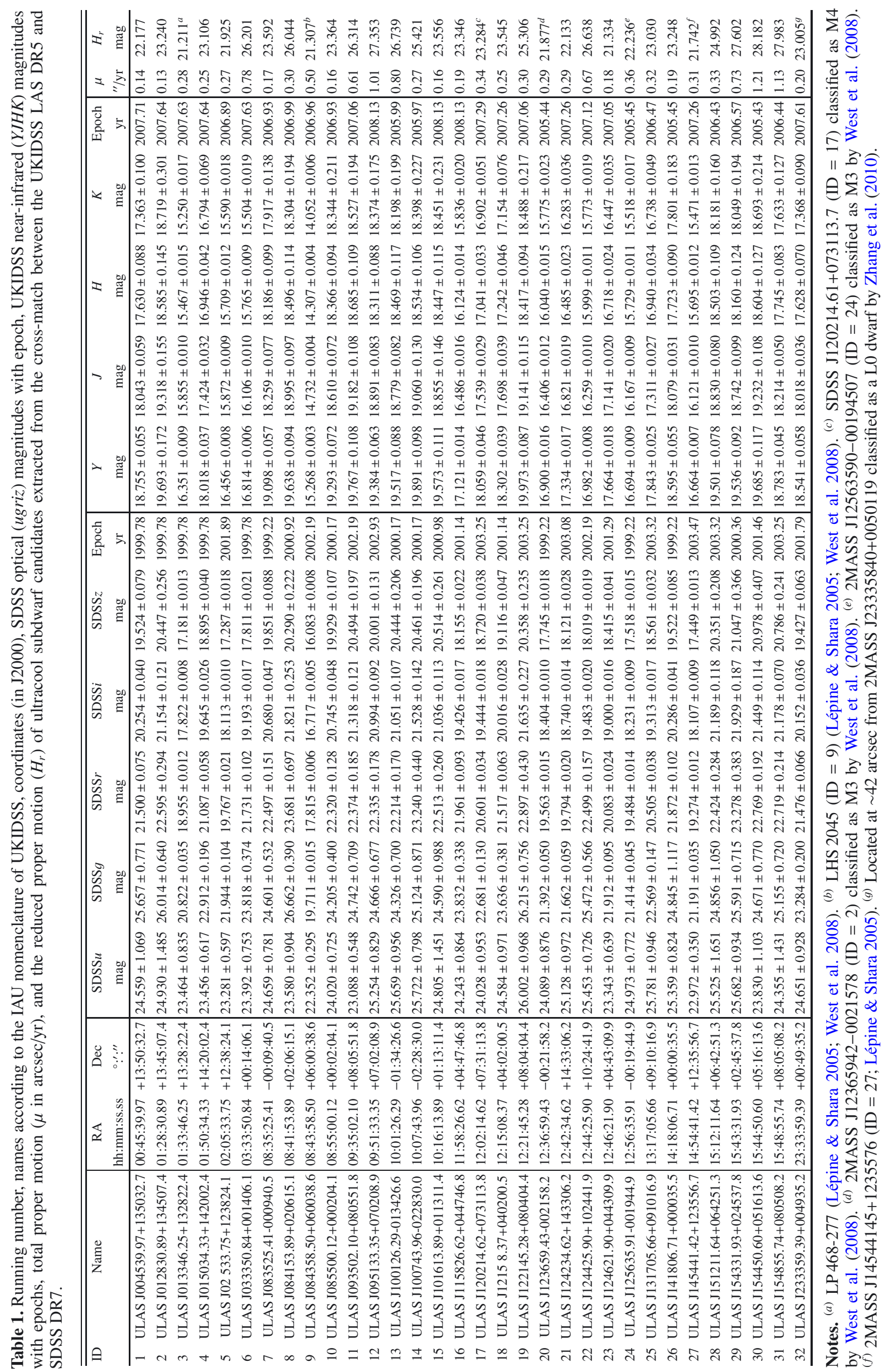


N. Lodieu et al.: Ultracool subdwarfs in large-scale surveys using VO tools. I.

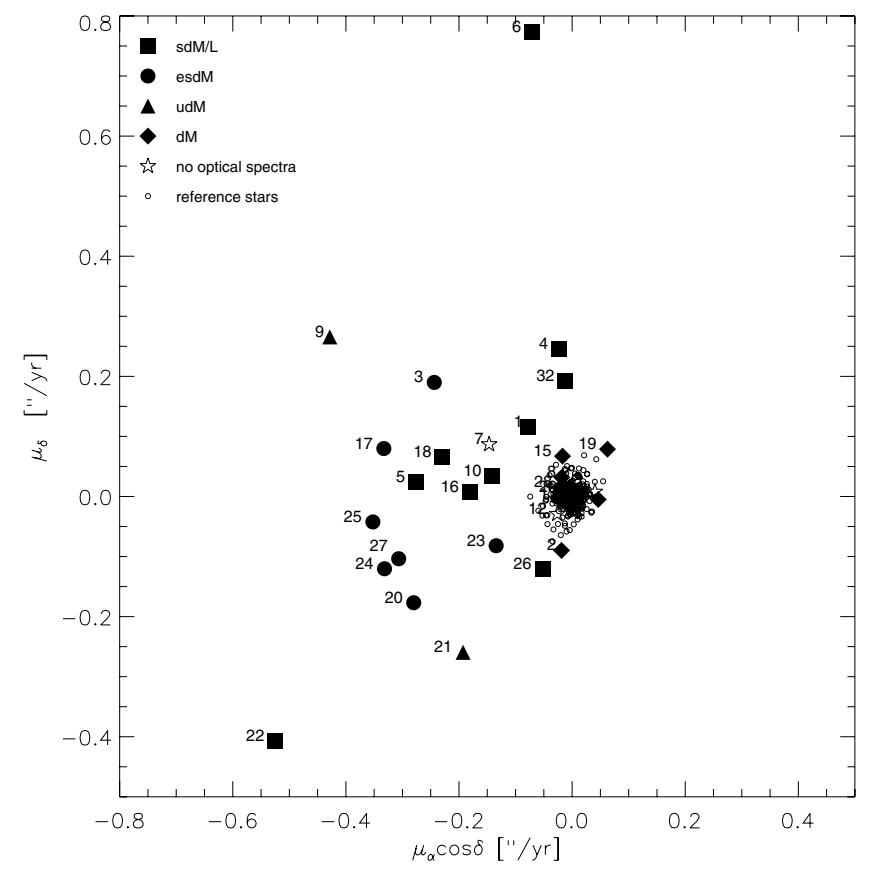

Fig. 3. Revised proper motions of our candidates. Astrometric reference stars (small open squares) are concentrated around $(0,0)$. Symbols as in Fig. 2. Error bars are smaller than the size of the symbols.

\subsection{Method}

We computed accurate proper motions by measuring the pixel coordinates $(x, y)$ of the targets and tens of other sources on the Sloan $z$ and UKIDSS LAS $J$ images. We carried out this procedure under the IRAF environment. Firstly, we downloaded the SDSS and UKIDSS LAS images with a size of 6 arcmin aside, centred on each target. Secondly, we identified high-quality point sources with a signal-to-noise ratio higher than 10 in both images, objects selected as reference stars for proper motion measurements. We assumed that these sources (about 30 per target) are not moving, which is a valid assumption because they are centred around $(0,0)$ in Fig. 3.

Then, we transformed the pixel coordinates from one epoch to the other using second-order polynomial transformations for the $x$ and $y$ axes. The dispersion of the transformations was typically 0.16 pixel (or 0.032 arcsec). The resulting $x$ and $y$ shifts were converted into proper motions by taking into account the time baseline of the data and the appropriate pixel scale values. Our measurements along their associated error bars are given in Table 2.

\subsection{New proper motions}

We find that the revised proper motions agree with the proper motion obtained from the VO for 22 of the 32 candidates $(\sim 70 \%)$ (Table 2) Below we give details on the 10 sources whose new proper motion differ from the original ones, leading to a different position in the reduced proper motion diagram (Table 2; Fig. 2):

- sources \#30 and \#31 should be rejected because no object is detected in the UKIDSS images, suggesting that these are false cross-matches. No optical spectra (Sect. 4) are available for these sources, therefore we rejected them from our sample;

- sources \#11 and \#12 have smaller proper motion because of large offsets between the position of the source on the
Table 2. Revised proper motions and reduced proper motions along with the values derived from the VO cross-match.

\begin{tabular}{|c|c|c|c|c|c|c|}
\hline ID & $\mu_{\alpha} \cos \delta$ & $\mu_{\delta}$ & $\mu_{\text {total }}$ & $\mathrm{Hr}$ & $\mu(\mathrm{VO})$ & $\operatorname{Hr}(\mathrm{VO})$ \\
\hline & $" \prime / y r$ & $1 / \mathrm{yr}$ & $" 1 / \mathrm{yr}$ & mag & $" 1 / \mathrm{yr}$ & mag \\
\hline 1 & $-0.077 \pm 0.005$ & $0.115 \pm 0.005$ & $0.139 \pm 0.007$ & 22.215 & 0.137 & 22.177 \\
\hline 2 & $0.004 \pm 0.005$ & $-0.103 \pm 0.005$ & $0.103 \pm 0.007$ & 22.659 & 0.135 & 23.240 \\
\hline 3 & $-0.244 \pm 0.001$ & $0.190 \pm 0.002$ & $0.309 \pm 0.002$ & 21.405 & 0.283 & 21.211 \\
\hline 4 & $-0.022 \pm 0.005$ & $0.246 \pm 0.004$ & $0.247 \pm 0.006$ & 23.050 & 0.253 & 23.106 \\
\hline 5 & $-0.276 \pm 0.002$ & $0.024 \pm 0.001$ & $0.277 \pm 0.002$ & 21.979 & 0.270 & 21.925 \\
\hline 6 & $-0.070 \pm 0.002$ & $0.773 \pm 0.001$ & $0.776 \pm 0.003$ & 26.180 & 0.783 & 26.201 \\
\hline 7 & $-0.147 \pm 0.003$ & $0.087 \pm 0.004$ & $0.171 \pm 0.005$ & 23.662 & 0.166 & 23.592 \\
\hline 8 & $-0.018 \pm 0.006$ & $0.032 \pm 0.006$ & $0.037 \pm 0.009$ & 21.522 & 0.297 & 26.044 \\
\hline 9 & $-0.429 \pm 0.006$ & $0.266 \pm 0.005$ & $0.504 \pm 0.008$ & 21.327 & 0.500 & 22.514 \\
\hline 10 & $-0.141 \pm 0.006$ & $0.034 \pm 0.006$ & $0.145 \pm 0.008$ & 23.127 & 0.162 & 23.364 \\
\hline 11 & $.040 \pm 0.007$ & $0.008 \pm 0.008$ & & 20.438 & 0.614 & 26.314 \\
\hline 12 & $-0.026 \pm 0.006$ & $-0.029 \pm 0.006$ & $0.039 \pm 0.008$ & 20.290 & 1.008 & 27.353 \\
\hline 13 & $0.103 \pm 0.007$ & $0.069 \pm 0.006$ & $0.124 \pm 0.009$ & 22.681 & 0.803 & 26.739 \\
\hline 14 & $0.046 \pm 0.006$ & $-0.005 \pm 0.006$ & $0.046 \pm 0.008$ & 21.554 & 0.273 & 25.421 \\
\hline 15 & $-0.017 \pm 0.005$ & $0.067 \pm 0$ & $0.069 \pm 0$ & 21.707 & 0.162 & 23.556 \\
\hline 16 & $-0.181 \pm 0.003$ & $0.008 \pm 0.005$ & $0.181 \pm 0.006$ & 23.249 & 0.189 & 23.346 \\
\hline 17 & $-0.332 \pm 0.007$ & $0.080 \pm 0.007$ & $0.341 \pm 0.010$ & 23.265 & 0.344 & 23.284 \\
\hline 18 & $-0.229 \pm 0.003$ & $0.066 \pm 0.003$ & $0.239 \pm 0$. & 23.409 & 0.255 & 23.545 \\
\hline 19 & $0.063 \pm 0.010$ & $0.080 \pm 0.010$ & $0.101 \pm 0.141$ & 22.919 & 0.303 & 25.306 \\
\hline 20 & $-0.280 \pm 0.004$ & $-0.177 \pm 0.003$ & $0.331 \pm 0.005$ & 22.162 & 0.290 & 21.877 \\
\hline 21 & $-0.193 \pm 0.007$ & $-0.259 \pm 0.008$ & $0.323 \pm 0.011$ & 22.340 & 0.294 & 22.133 \\
\hline 22 & $-0.525 \pm 0.007$ & $-0.407 \pm 0.007$ & $0.664 \pm 0.010$ & 26.610 & 0.673 & 26.638 \\
\hline 23 & $-0.134 \pm 0.005$ & $-0.082 \pm 0.004$ & $0.157 \pm 0.007$ & 21.062 & 0.178 & 21.334 \\
\hline 24 & $-0.332 \pm 0.006$ & $-0.120 \pm 0.006$ & $0.353 \pm 0.009$ & 22.223 & 0.355 & 22.236 \\
\hline 25 & $-0.352 \pm 0.010$ & $-0.042 \pm 0.012$ & $0.355 \pm 0.016$ & 23.256 & 0.320 & 23.030 \\
\hline 26 & $-0.052 \pm 0.003$ & $-0.121 \pm 0.004$ & $0.132 \pm 0.005$ & 22.475 & 0.188 & 23.248 \\
\hline 27 & $-0.307 \pm 0.010$ & $-0.104 \pm 0.010$ & $0.324 \pm 0.014$ & 21.827 & 0.312 & 21.742 \\
\hline 28 & $-0.016 \pm 0.008$ & $0.015 \pm 0.010$ & $0.022 \pm 0.013$ & 19.136 & 0.326 & 24.992 \\
\hline 29 & $-0.007 \pm 0.006$ & $0.008 \pm 0.006$ & $0.011 \pm 0.009$ & 18.485 & 0.733 & 27.602 \\
\hline 30 & - \pm- & - \pm- & - \pm- & - & 1.210 & 28.182 \\
\hline 31 & - \pm- & - \pm- & - \pm- & - & 1.129 & 27.983 \\
\hline 32 & $-0.012 \pm 0.004$ & $0.193 \pm 0.005$ & $0.193 \pm 0.007$ & 22.904 & 0.202 & 23.005 \\
\hline
\end{tabular}

SDSS image and the position reported by the SDSS catalogue. Their revised $\mathrm{Hr}$ is smaller than 20.7 mag, implying that they would not enter our sample. No optical spectra are available for them, therefore we reject them;

- three sources classified as M dwarfs (\#8, 28, and 29) were rejected because their revised proper motions are smaller than the one derived by the VO, leading to a lower $\mathrm{Hr}$ value and/or a position in the reduced proper motion diagram, suggesting that they are solar-metallicity dwarfs;

- three M dwarfs (\#14, 15, and 19) have smaller revised proper motions but lie in the same region as subdwarfs in the reduced proper motion diagram.

We note that sources \#7 and \#2 have revised proper motions identical to those from the VO and both objects lie in the subdwarf domain in the reduced proper motion diagram. Source \#7 has no optical spectroscopy, it thus remains a reliable ultracool subdwarf candidate. In contrast, source \#2 has optical spectroscopy, suggesting that it is a solar-metallicity $M$ dwarf that contaminates our sample.

To summarise, we conclude that only one of the five sources without optical spectrum remains as a bona-fide subdwarf. Moreover, three of the seven sources spectroscopically classified as M dwarfs (Fig. 6) would be rejected based on the revised proper motion, the remaining ones are photometrically contaminated because they lie in the region of the reduced proper motion diagram where ultracool subdwarfs are found (right panel in Fig. 2). 
Table 3. Log of the spectroscopic observations.

\begin{tabular}{|c|c|c|c|c|c|c|c|}
\hline $\begin{array}{l}\text { RA } \\
\text { hh:mm:ss.ss }\end{array}$ & $\begin{array}{l}\operatorname{Dec} \\
\circ::^{\prime \prime}\end{array}$ & Instr & $\begin{array}{c}\text { Date } \\
\text { yyyy-mm-dd }\end{array}$ & \# & $\begin{array}{c}\text { ExpT } \\
\text { sec }\end{array}$ & Airm & $\begin{array}{c}\text { Seeing } \\
\prime \prime\end{array}$ \\
\hline $00: 45: 39.97$ & $+13: 50: 32.7$ & FORS & $2009-11-10$ & 3 & 1170 & 1.286 & 0.74 \\
\hline $01: 28: 30.89$ & $+13: 45: 07.4$ & FORS & 2009-11-04/15 & 4 & 2640 & 1.286 & 0.62 \\
\hline $01: 50: 34.33$ & $+14: 20: 02.4$ & FORS & 2009-10-30 & 3 & 1170 & 1.494 & 0.59 \\
\hline $03: 33: 50.84$ & $+00: 14: 06.1$ & FORS & $2009-10-30$ & 3 & 870 & 1.546 & 0.80 \\
\hline $08: 41: 53.89$ & $+02: 06: 15.1$ & FORS & $2009-12-21 / 25$ & 4 & 2640 & 1.121 & 0.81 \\
\hline 08:55:00.12 & $+00: 02: 04.1$ & FORS & $2009-12-25$ & 1 & 660 & 1.122 & 0.65 \\
\hline $10: 01: 26.29$ & $-01: 34: 26.6$ & FORS & 2010-01-08 & 2 & 1330 & 1.092 & 0.86 \\
\hline $10: 07: 43.96$ & $-02: 28: 30.0$ & FORS & $2009-12-17 / 24$ & 4 & 2644 & 1.150 & 0.83 \\
\hline $10: 16: 13.89$ & $+01: 13: 11.4$ & FORS & $2009-12-24$ & 2 & 1320 & 1.161 & 0.56 \\
\hline $11: 58: 26.62$ & $+04: 47: 46.8$ & FORS & $2009-12-21$ & 1 & 1320 & 1.468 & 0.73 \\
\hline $12: 02: 14.62$ & $+07: 31: 13.8$ & FORS & $2010-01-23$ & 1 & 390 & 1.222 & 0.77 \\
\hline $12: 15: 08.37$ & $+04: 02: 00.5$ & FORS & 2010-02-12 & 3 & 1170 & 1.183 & 0.87 \\
\hline $12: 21: 45.28$ & $+08: 04: 04.4$ & FORS & $2010-01-08 / 09$ & 4 & 2644 & 1.280 & 0.60 \\
\hline $12: 42: 34.62$ & $+14: 33: 06.2$ & FORS & 2010-03-07 & 1 & 390 & 1.306 & 0.53 \\
\hline $12: 44: 25.90$ & $+10: 24: 41.9$ & FORS & 2010-01-23 & 1 & 390 & 1.237 & 0.95 \\
\hline $12: 46: 21.90$ & $+04: 43: 09.9$ & FORS & 2010-03-07 & 1 & 390 & 1.181 & 0.57 \\
\hline $13: 17: 05.66$ & $+09: 10: 16.9$ & FORS & 2010-03-07 & 1 & 390 & 1.239 & 0.52 \\
\hline $14: 18: 06.71$ & $+00: 00: 35.5$ & FORS & $2010-02-13$ & 3 & 1170 & 1.119 & 0.71 \\
\hline $15: 12: 11.64$ & $+06: 42: 51.3$ & FORS & 2010-03-07 & 4 & 2640 & 1.510 & 0.57 \\
\hline $15: 43: 31.93$ & $+02: 45: 37.8$ & FORS & 2010-03-07 & 8 & 5280 & 1.333 & 0.63 \\
\hline $23: 33: 59.39$ & $+00: 49: 35.2$ & FORS & 2009-11-10 & 3 & 1170 & 1.130 & 1.14 \\
\hline $14: 54: 41.42$ & $+12: 35: 56.7$ & NOT & 2011-09-03 & 3 & 2400 & 1.700 & 0.80 \\
\hline $01: 33: 46.25$ & $+13: 28: 22.4$ & SDSS & $2000-12-01$ & 5 & 5400 & 1.143 & 2.82 \\
\hline $02: 05: 33.75$ & $+12: 38: 24.1$ & SDSS & $2000-12-01$ & 5 & 4500 & 1.143 & 2.82 \\
\hline $08: 43: 58.50$ & $+06: 00: 38.6$ & SDSS & 2003-03-10 & 3 & 2400 & 1.161 & 1.81 \\
\hline $12: 02: 14.62$ & $+07: 31: 13.8$ & SDSS & 2004-03-25 & 3 & 3000 & 1.133 & 1.41 \\
\hline $12: 36: 59.43$ & $-00: 21: 58.2$ & SDSS & 2001-02-01 & 4 & 3600 & 1.209 & 2.87 \\
\hline $12: 56: 35.91$ & $-00: 19: 44.9$ & SDSS & $2001-03-26$ & 3 & 2702 & 1.214 & 2.28 \\
\hline $13: 17: 05.66$ & $+09: 10: 16.9$ & SDSS & 2006-04-25 & 4 & 4900 & 1.216 & 2.02 \\
\hline
\end{tabular}

\section{Optical spectroscopy}

We emphasise that optical spectroscopy was obtained for candidates identified in the preliminary search, i.e. with the proper motion derived from the $\mathrm{VO}$ and not from the revised proper motions discussed in Sect. 3.

\subsection{NOT spectroscopy}

We carried out low-resolution $(R \sim 450)$ optical $(500-1025 \mathrm{~nm})$ spectroscopy with the ALFOSC spectrograph on the $2.5-\mathrm{m}$ Nordic Optical Telescope (NOT) at the Observatory of the Roque de Los Muchachos on the island of La Palma, Canary Islands. One candidate, ULAS J145441.41+123556.6(ID = 27), was observed on 3 September 2011 (Table 3). Weather conditions were non-photometric with high level clouds and seeing around $0.7-0.9$ arcsec. The object was observed around UT = $21 \mathrm{~h}$ at parallactic angle under high airmass, starting at 1.5 and finishing around 1.9 (Table 3).

The ALFOSC spectrograph is equipped with a $2048 \times$ 2052 pixel back-illuminated CCD42-40 charge-coupled device. We employed grism number 5. The total exposure time for ULAS J145441.41+123556.6 was divided into three on-source integrations of $800 \mathrm{~s}$ with a slit width of 1 arcsec to achieve a resolution of $1.55 \mathrm{~nm}$ at $700 \mathrm{~nm}$. An internal flat-field was obtained immediately after the target to remove the fringing at long wavelength as much as possible, the effect being of the order of $18 \%$ at $800 \mathrm{~nm}$. Unfortunately, fringing is clearly visible beyond $800 \mathrm{~nm}$ because the internal flat field was obtained after the last of the three individual exposures taken for the target. Bias frames were observed in the afternoon before the beginning of the night. $\mathrm{He}, \mathrm{Ne}$, and Ar arc lamps were also obtained immediately after each exposure to calibrate our target in wavelength.
The data reduction of the NOT/ALFOSC spectra was entirely carried out under the IRAF ${ }^{4}$ environment. First, we subtracted the average of all bias frames from the raw science exposures and then divided by the normalised response function of the mean flat field (also bias-subtracted). Second, we extracted a one-dimensional spectrum interactively by choosing the appropriate width for the aperture width. Then, we used the arc lamps to calibrate our spectra in wavelength with an accuracy better than $0.2 \AA$. The flux calibration of the spectrum was conducted using BD+174708 (Latham et al. 2002) as spectrophotometric standard star. The final optical spectrum, covering the $500-900 \mathrm{~nm}$ wavelength range and normalised at $750 \mathrm{~nm}$, is shown in Fig. 5.

\subsection{FORS2 spectroscopy}

We conducted low-resolution $(R \sim 350)$ optical $(600-1010 \mathrm{~nm})$ spectroscopy with the visual and near-UV FOcal Reducer and low-dispersion Spectrograph FORS2 (Appenzeller et al. 1998) on the ESO VLT Antu unit in Paranal, Chile. We observed 22 subdwarf candidates identified in the UKIDSS LAS DR5 vs. SDSS DR7 cross-match (Table 3). All observations were conducted in service mode under programmes 084.C-0928A and 084.C-0928B. The requested conditions, grey time, thin cirrus acceptable, and seeing less than 1.4 and 1.0 arcsec for the bright and faint targets, respectively, were generally satisfied (Table 3). All objects were observed at parallactic angle.

The FORS2 instrument is equipped with two $2048 \times$ 4096 MIT CCDs with pixels of $15 \mu \mathrm{m}$ working in the 330-1100 nm range (Appenzeller et al. 1998). We employed the grism $150+27$ with the order-blocking filter OG590 with the standard resolution of $2.07 \mathrm{~nm}$ per pixel and a slit of 1.0 arcsec to achieve a spectral resolution of $\sim 175$ at $720 \mathrm{~nm}$ due to the $2 \times 2$ binning. The exposure time was scaled according to the brightness of the target in the Sloan $r$ and $i$ filter sin order to achieve a minimum signal-to-noise ratio of 20 . The faintest sources, observed several times along the slit, have a lower signal-to-noise ratio because they are faint even for a 8-m class telescope. Dome flat fields, bias frames, arc lamps, and spectrophotometric standard stars were observed every night as part of the ESO calibration plan.

As for NOT/ALFOSC, the data reduction of the VLT FORS2 spectra was entirely carried out under the IRAF environment. First, we cut the 2D images to select the interesting part of the spectrum, from $\sim 600$ to $1000 \mathrm{~nm}$ roughly. Then, we subtracted the average of all bias frames from the raw science exposures and from the median-combined dome flat. Afterwards, we divided the science frame by the normalised flat field using the mean value over the entire dome flat frame. Later, we extracted a one-dimensional spectrum interactively by choosing the appropriate value for the aperture width. Then, we used the arc lamps with helium, $\mathrm{HgCd}$, and argon to calibrate our spectra in wavelength with an accuracy about $0.4-0.6 \AA \mathrm{rms}$. Finally we calibrated the 1D spectra with spectrophotometric standard stars observed on the same night as the target. Spectra were normalised at $750 \mathrm{~nm}$ and were not corrected for the telluric band around $760 \mathrm{~nm}$. The VLT FORS2 spectra of the 11 candidates classified as subdwarfs are displayed in Fig. 4. The sources classified as extreme subdwarfs (seven objects) and ultra-subdwarf

\footnotetext{
4 IRAF is distributed by the National Optical Astronomy Observatory, which is operated by the Association of Universities for Research in Astronomy (AURA) under cooperative agreement with the National Science Foundation.
} 
N. Lodieu et al.: Ultracool subdwarfs in large-scale surveys using VO tools. I.

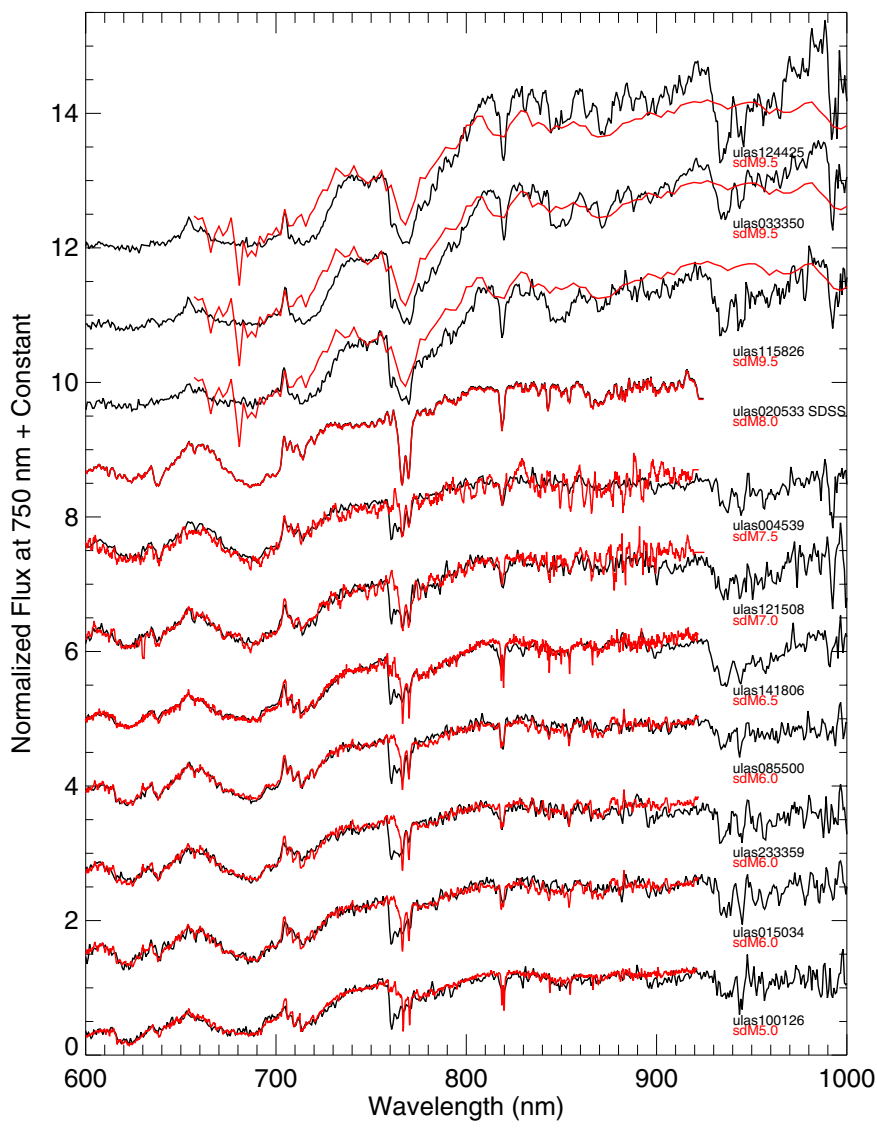

Fig. 4. Low-resolution optical (600-1000 nm) spectra of confirmed subdwarfs obtained with VLT FORS2. Spectra are ordered by increasing spectral type and offset by a constant value for clarity. Overplotted in red are known template subdwarfs from the SDSS spectroscopic database (600-940 nm) except for the top 3 templates which come from the IRTF/SpeX library.

(two objects) are shown in Fig. 5 while the seven sources classified as solar-metallicity $\mathrm{M}$ dwarfs are displayed in Fig. 6. The two known subdwarfs previously reported in the literature are included in these figures.

\subsection{SDSS spectroscopy}

The SDSS spectroscopic database represents an invaluable source of good-quality spectra for a large number of astronomical sources. These spectra, covering the 380-940 nm wavelength range with a resolution of $\sim 2000$ are publicly available from the Sloan webpage. They are wavelength- and fluxcalibrated and also corrected for telluric absorption bands. The total exposure times consist of several integrations of $900 \mathrm{~s}$. We found seven of our 32 ultracool subdwarf candidates in this database, two of them (ULAS J131705.66+091016.9 (ID = 25) and ULAS J120214.62+073113.8 (ID = 17)) re-observed with VLT FORS 2 because of their poorer quality (Table 3 ). The other five SDSS spectra have a good signal-to-noise ratio and we analysed them in a similar manner as the VLT FORS2 spectra (see Sect. 5). The observing date, number of exposures, airmass and best $80 \%$ seeing are listed at the bottom of Table 3 .

\section{New ultracool subdwarfs}

In this section, we assign spectral types to the new subdwarfs, measure their radial velocities, estimate their spectroscopic

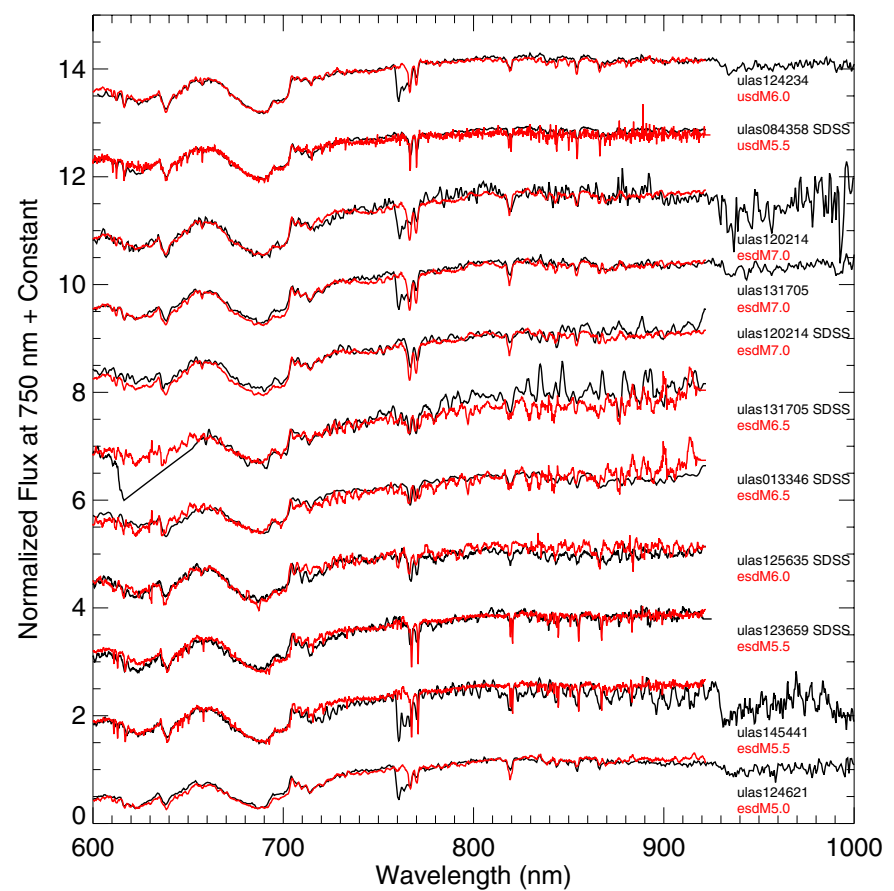

Fig. 5. Low-resolution optical (600-1000 nm) spectra of confirmed extreme subdwarfs and ultra-subdwarfs obtained with VLT FORS2. The second spectrum from the bottom is from NOT/ALFOSC. Spectra are ordered by increasing spectral type and offset by a constant value for clarity. Overplotted in red are known template extreme subdwarfs and ultra-subdwarfs from the SDSS spectroscopic database (600-940 nm).

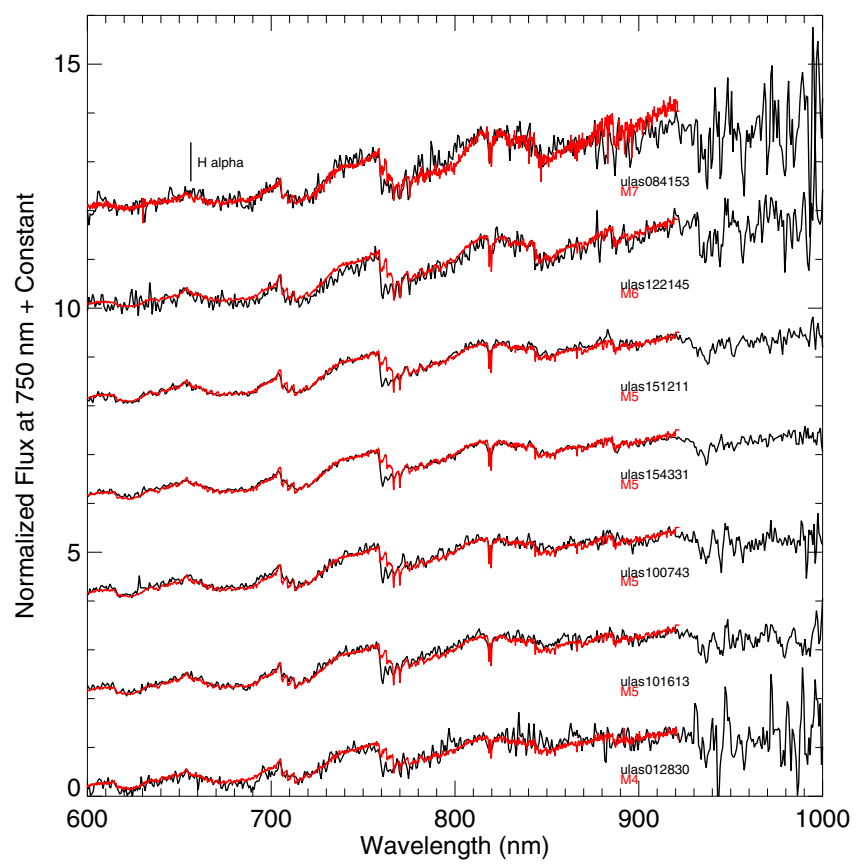

Fig. 6. Low-resolution optical (600-1000 nm) spectra of photometric candidates classified as solar-metallicity M dwarfs. Spectra are ordered by increasing spectral type and offset by a constant value for clarity. Overplotted in red are known M-dwarf templates downloaded from the SDSS spectroscopic database.

distances, discuss their mid-infrared properties, search for wide companions, and present a preliminary estimate of their surface density. 
Table 4. Coordinates (in J2000), spectral indices, spectral types determined following the definitions by Gizis (1997) and Lépine et al. (2007) for the new subdwarfs.

\begin{tabular}{|c|c|c|c|c|c|c|c|c|c|c|}
\hline$\overline{\mathrm{ID}}$ & $\begin{array}{c}\text { RA } \\
\text { hh:mm:ss.ss }\end{array}$ & $\begin{array}{l}\text { Dec } \\
0^{\prime}::^{\prime \prime}\end{array}$ & TiO5 & $\mathrm{CaH} 1$ & $\overline{\mathrm{CaH} 2}$ & $\mathrm{CaH3}$ & TiO5 & SpT_Gizis & SppT_Lepine & SpT_final \\
\hline 1 & $00: 45: 39.97$ & $+13: 50: 32.7$ & 0.715 & 0.477 & 0.297 & 0.465 & 0.176 & esdM5.2 & esdM5.6 & sdM7.5 \\
\hline 3 & $01: 33: 46.25$ & $+13: 28: 22.4$ & 0.911 & 0.340 & 0.321 & 0.468 & 0.194 & esdM5.2 & usdM5.4 & esdM6.5 \\
\hline 4 & $01: 50: 34.33$ & $+14: 20: 02.4$ & 0.656 & 0.552 & 0.402 & 0.579 & 0.324 & sdM4.4 & sdM3.9 & sdM6.0 \\
\hline 5 & $02: 05: 33.75$ & $+12: 38: 24.1$ & 0.648 & 0.270 & 0.141 & 0.268 & 0.002 & esdM7.9 & esdM8.5 & sdM8.0 \\
\hline 6 & 03:33:50.84 & $+00: 14: 06.1$ & 0.209 & 0.343 & 0.147 & 0.337 & 0.031 & sdM8.3 & sdM7.9 & sdL0.0 \\
\hline 9 & $08: 43: 58.50$ & $+06: 00: 38.6$ & 0.814 & 0.551 & 0.377 & 0.544 & 0.281 & sdM5.1 & esdM4.4 & usdM5.5 \\
\hline 10 & 08:55:00.12 & $+00: 02: 04.1$ & 0.546 & 0.600 & 0.280 & 0.463 & 0.165 & esdM5.3 & sdM5.7 & sdM6.0 \\
\hline 13 & 10:01:26.29 & $-01: 34: 26.6$ & 0.611 & 0.814 & 0.532 & 0.783 & 0.581 & $\mathrm{dM} 0.0$ & dM1.7 & sdM5.0 \\
\hline 16 & 11:58:26.62 & $+04: 47: 46.8$ & 0.140 & 0.816 & 0.218 & 0.387 & 0.088 & sdM7.5 & dM6.8 & sdM9.5 \\
\hline 17 & 12:02:14.62 & $+07: 31: 13.8$ & 0.959 & 0.314 & 0.258 & 0.439 & 0.137 & esdM5.6 & usdM6.1 & esdM7.0 \\
\hline 17 & 12:02:14.62 & $+07: 31: 13.8$ & 0.877 & 0.720 & 0.401 & 0.511 & 0.275 & esdM4.6 & usdM4.4 & esdM7.0 \\
\hline 18 & $12: 15: 08.37$ & $+04: 02: 00.5$ & 0.528 & 0.628 & 0.218 & 0.405 & 0.097 & esdM6.0 & sdM6.7 & sdM7.0 \\
\hline 20 & $12: 36: 59.43$ & $-00: 21: 58.2$ & 0.693 & 0.609 & 0.343 & 0.558 & 0.268 & esdM4.0 & esdM4.5 & esdM5.5 \\
\hline 21 & $12: 42: 34.62$ & $+14: 33: 06.2$ & 0.985 & 0.556 & 0.342 & 0.521 & 0.242 & esdM4.5 & usdM4.8 & usdM5.0 \\
\hline 22 & $12: 44: 25.90$ & $+10: 24: 41.9$ & 0.135 & 0.657 & 0.180 & 0.272 & 0.018 & sdM9.4 & dM8.2 & sdL0.5 \\
\hline 23 & $12: 46: 21.90$ & $+04: 43: 09.9$ & 0.702 & 0.688 & 0.421 & 0.625 & 0.372 & sdM3.7 & esdM3.5 & esdM5.0 \\
\hline 24 & $12: 56: 35.91$ & $-00: 19: 44.9$ & 0.722 & 0.597 & 0.347 & 0.483 & 0.220 & esdM5.0 & esdM5.1 & esdM6.0 \\
\hline 25 & $13: 17: 05.66$ & $+09: 10: 16.9$ & 0.726 & 0.496 & 0.319 & 0.481 & 0.201 & esdM5.0 & esdM5.3 & esdM7.0 \\
\hline 25 & $13: 17: 05.66$ & $+09: 10: 16.9$ & 0.641 & 1.079 & 0.240 & 0.434 & 0.125 & esdM5.7 & esdM6.3 & esdM6.5 \\
\hline 26 & $14: 18: 06.71$ & $+00: 00: 35.5$ & 0.421 & 0.702 & 0.330 & 0.565 & 0.264 & sdM4.6 & sdM4.6 & sdM6.5 \\
\hline 27 & $14: 54: 41.42$ & $+12: 35: 56.7$ & 0.742 & 0.348 & 0.499 & 0.633 & 0.231 & esdM4.8 & esdM4.9 & esdM5.5 \\
\hline 32 & $23: 33: 59.39$ & $+00: 49: 35.2$ & 0.706 & 0.603 & 0.328 & 0.523 & 0.233 & esdM4.5 & esdM4.9 & sdM6.0 \\
\hline
\end{tabular}

Notes. The last column gives the adopted spectral types derived from direct comparison with spectral templates. If a target appears twice, the first line corresponds to the FORS2 spectrum, while the second is the SDSS spectrum.

\subsection{Spectral types}

We employed two independent, but complementary methods to assign spectral types to our new ultracool subdwarfs.

The adopted classification for M-type subdwarfs and extreme subdwarfs relies on the scheme proposed by Gizis (1997) and extends to spectral types sdM7 and esdM5.5. This scheme is based on the strength of $\mathrm{CaH}$ (temperature index) and $\mathrm{TiO}$ (metallicity index) bands. An extension to later spectral types (up to sdM9) has been more recently proposed by Lépine et al. (2003a).

We measured the four spectral indices ( $\mathrm{TiO} 5, \mathrm{CaH} 1, \mathrm{CaH} 2$, and $\mathrm{CaH} 3$ ) defined by Gizis (1997) and later updated by Lépine et al. (2007) to distinguish ultra-subdwarfs, extreme subdwarfs, and subdwarfs from solar-metallicity $\mathrm{M}$ dwarfs based on the strength of the $\mathrm{CaH}$ and $\mathrm{TiO}$ absorption bands (Fig. 7). The spectral indices and their associated spectral types (quoted to the nearest decimal) derived for each of the confirmed subdwarfs are listed in Table 4 and plotted in Figs. 7,8.

The SDSS spectroscopic database provides optical spectra over the 600-940 nm wavelength range for a large number of metal-poor dwarfs with spectral types based on two independent classification schemes: the Hammer scheme discussed by Covey et al. (2007) and the updated subdwarf classification proposed by Lépine et al. (2007). The former distinguishes between solar-metallicity M dwarfs and subdwarfs, whereas the latter provides an accurate classification for metal-poor dwarfs with subclasses. Therefore, we downloaded from the Sloan spectroscopic database the brightest object of each subclass between 0 and 9 for subdwarfs, extreme subdwarfs, and ultra-subdwarfs. These "templates" were used to visually assign spectral types to our targets with an uncertainty of half a subclass (or better). We should mention that the SDSS templates were corrected for telluric absorption, whereas our spectra were not, resulting in a difference between them around the position of the $\mathrm{O}_{2}$ telluric line of about $760 \mathrm{~nm}$. We note that the sdM9.5 template was taken from the SpeX library ${ }^{5}$ because no sdM9.5 template was found in the SDSS spectroscopic database. The resulting fits (red) to our spectra (black) are presented in Figs. 4 to 6 .

The comparison between the spectral types inferred from spectral indices and from the direct comparison with "templates" is shown in Fig. 9. We find that the spectral types derived from spectral indices tend to under-estimate the spectral type (overestimate the effective temperature). Therefore, we adopted the direct and visual comparison with "templates" to assign spectral types to our targets because it provides a more accurate classification. We note that the spectral indices are not so reliable to classify subdwarfs because they rely on a narrow wavelength range, as pointed out by Lépine et al. (2007), and they also depend strongly on the resolution of the spectra (Table 4). The differences between both classifications are listed in Table 4 and are shown in Fig. 9, where asterisks represent confirmed metal-poor dwarfs with discrepant classes (not only spectral types) derived from the spectral indices and the direct comparison with templates, suggesting some shortcomings in the spectral types derived solely on indices. Our sample of 20 low-mass stars with low-metal content consists of nine subdwarfs, seven extreme subdwarfs, two ultra-subdwarfs, and two L subdwarfs.

The remaining seven sources in our spectroscopic sample are M dwarfs with spectral types ranging from M4 to M7. The contamination of our sample by these M dwarfs probably comes from large error bars on their Sloan positions, which leads to spurious proper motions and places them at the bottom of the reduced proper motion diagram where they mimick the subdwarf candidates. We remark that a revision of proper motions as outlined in Sect. 3 likely reduces the contamination level by a factor of 2-3. None of these M dwarfs exhibits $\mathrm{H} \alpha$ in emission at the resolution of our spectra $(R \sim 150)$, suggesting that

5 http://web.mit.edu/ajb/www/browndwarfs/spexprism/ 
N. Lodieu et al.: Ultracool subdwarfs in large-scale surveys using VO tools. I.
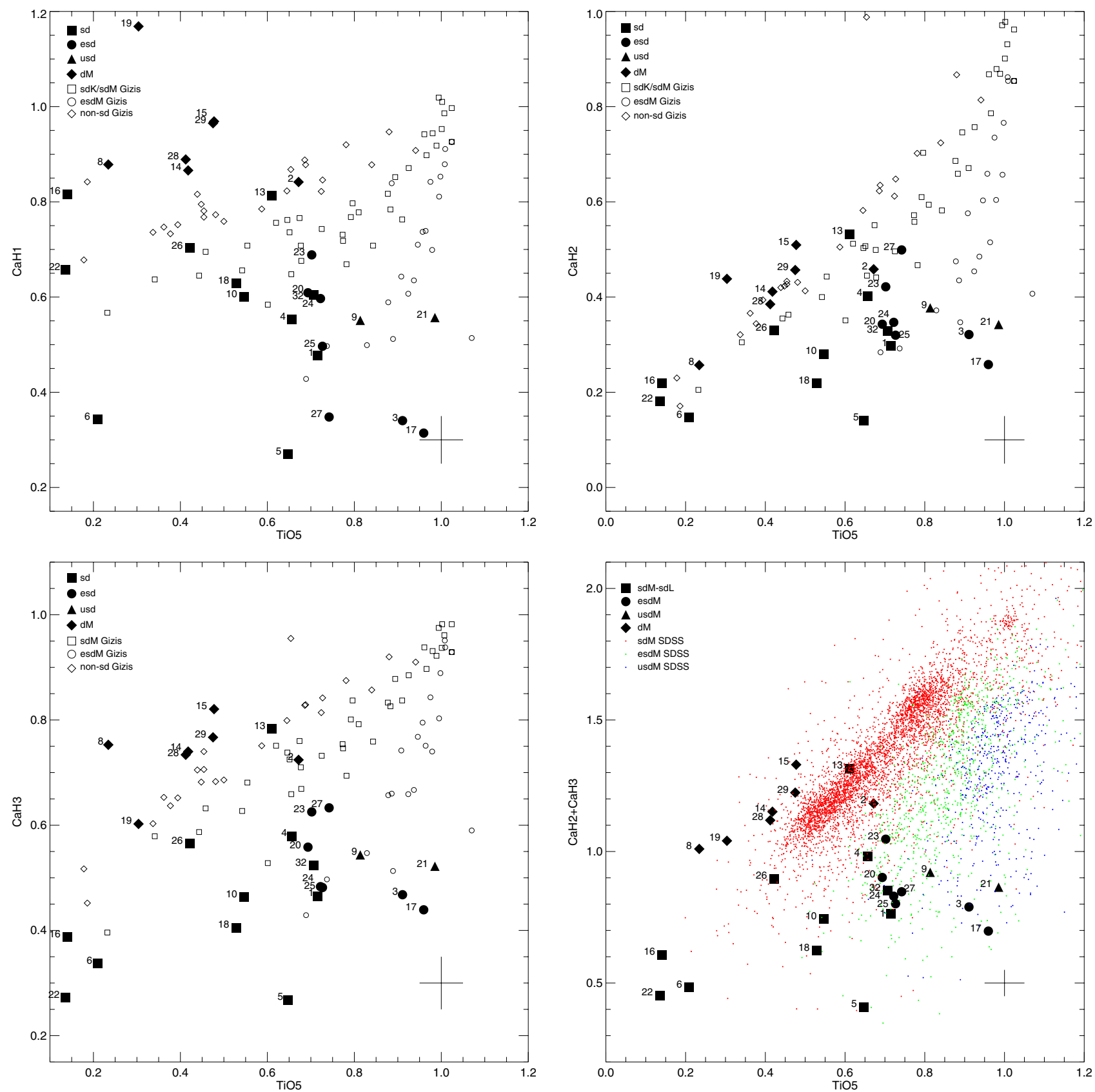

Fig. 7. Top left: $\mathrm{CaH} 1$ vs. TiO5 indices for our new ultracool subdwarfs. Top right: $\mathrm{CaH} 2$ vs. TiO5 indices. Bottom left: $\mathrm{CaH} 3$ vs. TiO5 indices. Bottom right: sum of $\mathrm{CaH} 2$ and $\mathrm{CaH} 3$ vs. TiO5 indices. Subdwarfs, extreme subdwarfs, ultra-subdwarfs, old solar-metallicity M dwarfs from our sample are marked as filled squares, circles, triangles, and diamonds, respectively. Numbers denominating our new discoveries follow the order by right ascension from Table 4. Open symbols are known subdwarfs discussed in Gizis (1997). The small coloured dots in the bottom right plot represent sources with SDSS spectroscopy classified as subdwarfs (red), extreme subdwarfs (green), and ultra-subdwarfs (blue). For the subdwarfs with two spectra from FORS2 and SDSS, we plotted only the indices derived from the VLT FORS2 spectra. Typical uncertainties on the spectral indices are about of 0.1 (cross at the bottom of each plot). These plots follow the standard figures presented in Fig. 1 of Gizis (1997) and Fig. 3 of Lépine et al. (2007).

they are older than typical $\mathrm{M}$ dwarfs in the solar vicinity. West et al. (2008) reported $\mathrm{H} \alpha$ in emission for $50 \%$ of the sources or more at spectral types later than M4, with equivalent widths broader than $1 \AA$. These authors also discussed the decrease in activity with age and scale height, suggesting that the $\mathrm{M}$ dwarfs that contaminate our sample may be older than the average lowmass stars in the solar neighbourhood and may be located at a higher scale height. The lack of $\mathrm{H} \alpha$ in emission also places a lower limit on the ages of these M dwarfs, 5 and 8 Gyr for M5 and M7 dwarfs.

\subsection{Radial velocities}

In this section we tentatively compute the radial velocities of our targets in spite of the low-resolution of our spectra. We used two methods.

First, we computed the offsets between lines resolved in our FORS2 spectra and the centroids of several atomic lines whose accurate positions can be found on the webpage of the National Institute of Standards and Technology ${ }^{6}$ : Ca I at $6572.18 \AA$, Ti I

${ }^{6}$ http://physics.nist.gov/asd3 

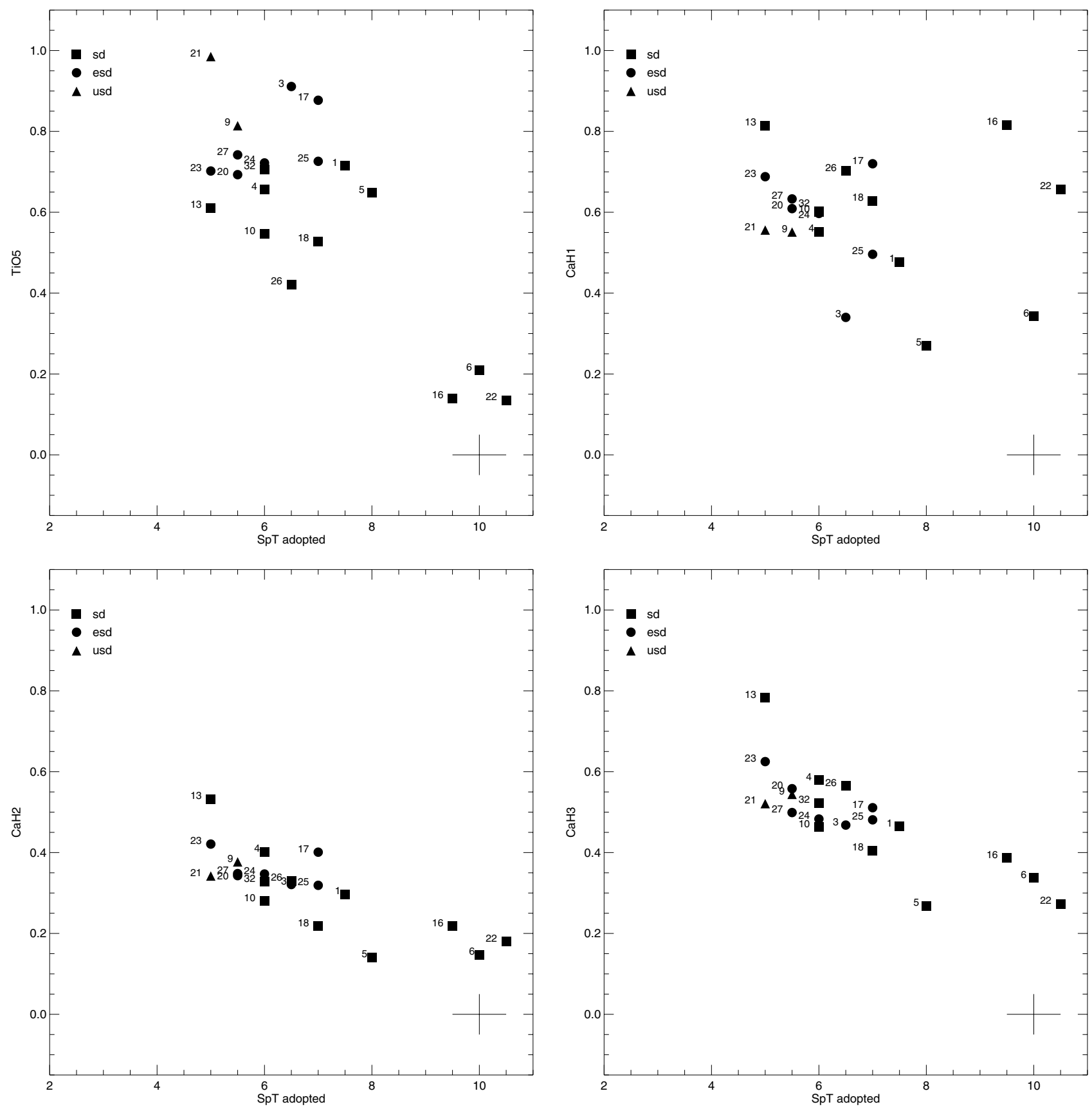

Fig. 8. Spectral indices as a function of the adopted spectral types for our new ultracool subdwarfs. Typical uncertainties on the spectral indices are of the order of 0.1 (cross at the bottom of each plot). Uncertainties on the spectral types are 0.5 subclass. Top left: adopted spectral types vs. TiO5 index. Top right: adopted spectral types vs. CaH1 index. Bottom left: adopted spectral types vs. CaH2 index. Bottom right: adopted spectral types vs. $\mathrm{CaH} 3$ index.

at $8434.94 \AA$, Na I doublet at 8183.25 and $8194.79 \AA$, Cs I at 8542.09 and $8943.47 \AA$, and Ca II at $8542.09 \AA$ (see also Table 2 of Burgasser et al. 2009). The uncertainties on these offsets are typically about $1 \AA$, corresponding to typical uncertainties of $35-50 \mathrm{~km} \mathrm{~s}^{-1}$. We focused only on the CaI, NaI doublet, and Ca II lines for the NOT spectrum and the SDSS spectra. We derived the observed radial velocities by multiplying those offsets by the speed of light and dividing by the wavelength $\left(c^{*} \Delta \lambda / \lambda\right)$. Observed velocities were converted into heliocentric velocities by computing the Earth's rotation, the motion of the Earth's center about the Earth-Moon barycentre, and the motion of the Earth-Moon barycentre about the centre of the Sun using the RVCORRECT routine in IRAF. In the fifth column of Table 5 we provide our heliocentric radial velocities measured with this method.

Independently, we computed radial velocities by crosscorrelating our optical spectra with the optical spectrum of the sdL3.5 subdwarf SDSS J125637.13-022452.4 (Burgasser et al. 2009) using the task FXCOR under the IRAF environment. This subdwarf has a well-measured radial velocity of $-130 \mathrm{~km} \mathrm{~s}^{-1}$ and we decided to use it as a template although it has a spectral type later than our confirmed subdwarfs. We transformed the vacuum wavelength published by Burgasser et al. (2009) into air wavelength and degraded its resolution to match our observations. We cross-matched the full spectrum of our sdM9.5 and sdL subdwarfs with Burgasser's template 


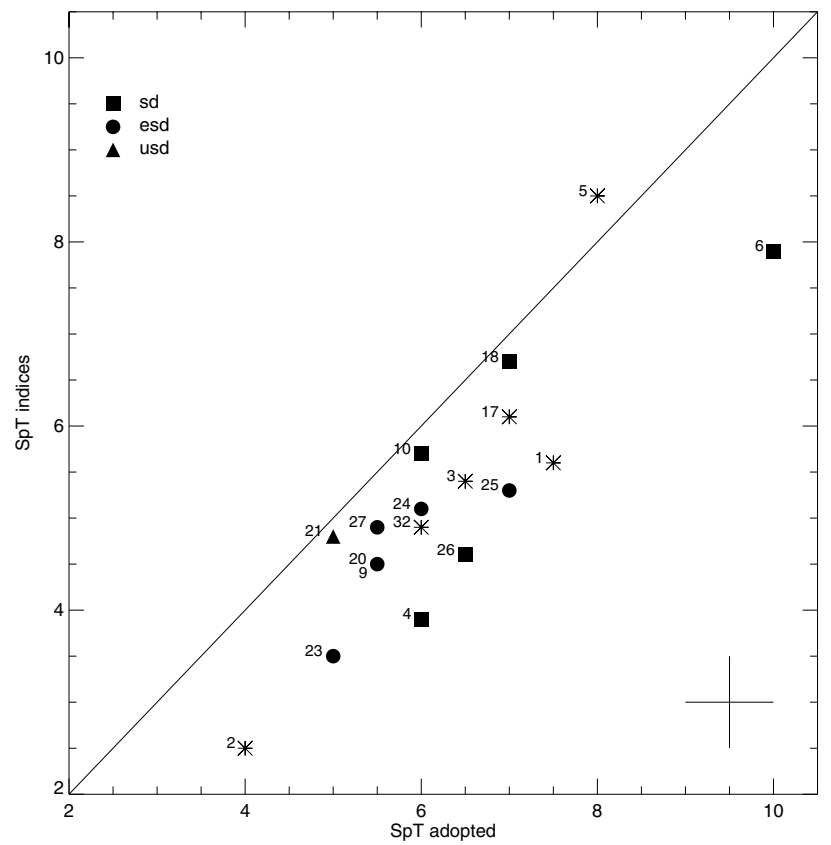

Fig. 9. Comparison of the spectral types obtained from the visual comparison with metal-poor "templates" and spectral types derived from the spectral indices defined by Gizis (1997) and Lépine et al. (2007). Subdwarfs, extreme subdwarfs, and ultra-subdwarfs are marked as squares, circles, and triangles, respectively. Metal-poor dwarfs with discrepant spectral types derived from both classification methods (comparison with templates vs. spectral indices) are displayed with asterisks (e.g. sdM vs. esdM or usdM vs. esdM, etc.). Typical uncertainties on the spectral types derived from both methods are half a subclass.

because of the similarities in the shape of the spectra and spectral types, but focused only on the NaI doublet region for the earlier subdwarfs in our sample. We cross-matched the spectra using two different functions: a Gaussian fit to the crosscorrelation function peak or simply the central value of the peak. Both methods led to differences smaller than the uncertainties output by FXCOR. The radial velocities inferred by this method are listed in Table 5 and have typical uncertainties of $40-50 \mathrm{~km} \mathrm{~s}^{-1}$ measured from the dispersion of the measurements from different parts of the spectra. Radial velocities derived from both methods usually agree within the uncertainties, except for ULAS J115826.62+044746.8 (ID = 16) and ULAS J123659.43-002158.2. In this case, we favour the radial velocities derived from the direct comparison with SDSS J125637.13022452.4 .

\subsection{Spectroscopic distances}

In this section, we estimate the spectroscopic distances by comparing our new discoveries with subdwarfs of similar spectral types with known trigonometric parallaxes.

We looked for subdwarfs with parallaxes whose spectral types are sdM5, sdM6, sdM6.5, sdM7, sdM7.5, and sdM9.5. We considered the following sources as subdwarfs with known distances to derive spectroscopic distances for our new ultracool subdwarfs: LP 807-23 (sdM5.0; $J=12.92$ mag; $d=$ 28.17-31.74 pc; van Altena et al. 1995), LHS 1074 (sdM6; $J=14.68 \mathrm{mag} ; d=85.7 \pm 17.1 \mathrm{pc} ;$ Salim \& Gould 2003; Riaz et al. 2008), LHS 1166 (sdM6.5; $J=14.26$ mag; $d=73-89$ pc; van Altena et al. 1995), LP 440-52 (sdM7; $J=13.19 \mathrm{mag} ; d=34.4-36.1 \mathrm{pc}$; van Altena et al. 1995), LSR J203621.86+505950.3 (sdM7.5; $J=13.628 \mathrm{mag}$;
Table 5. Coordinates (in J2000), spectral types, and radial velocities (expressed in $\mathrm{km} \mathrm{s}^{-1}$ ) derived in two different ways: first by measuring the offsets of well-known lines comparing with their centers (typical uncertainties of $35-50 \mathrm{~km} \mathrm{~s}^{-1}$ ) and, second, by cross-correlating the NaI doublet region of our optical spectra using SDSS J125637.13-022452.4 (sdL3.5) as template.

\begin{tabular}{cccccc}
\hline \hline ID & $\begin{array}{c}\text { RA } \\
\text { hh:mm:ss.ss }\end{array}$ & $\begin{array}{c}\text { Dec } \\
{ }^{\prime}::^{\prime \prime}\end{array}$ & SpT & $\begin{array}{c}V h_{1} \\
\mathrm{~km} \mathrm{~s}^{-1}\end{array}$ & $\begin{array}{c}V h_{2} \\
\mathrm{~km} \mathrm{~s}^{-1}\end{array}$ \\
\hline 1 & $00: 45: 39.97$ & $+13: 50: 32.7$ & sdM7.5 & 34.5 & 2.7 \\
3 & $01: 33: 46.25$ & $+13: 28: 22.4$ & esdM6.5 & 107.1 & 29.0 \\
4 & $01: 50: 34.33$ & $+14: 20: 02.4$ & sdM6.0 & 205.7 & 134.2 \\
5 & $02: 05: 33.75$ & $+12: 38: 24.1$ & sdM8.0 & -3.5 & - \\
6 & $03: 33: 50.84$ & $+00: 14: 06.1$ & sdL0.0 & 251.4 & 368.0 \\
9 & $08: 43: 58.50$ & $+06: 00: 38.6$ & usdM5.5 & 176.8 & 126.7 \\
10 & $08: 55: 00.12$ & $+00: 02: 04.1$ & sdM6.0 & 69.7 & -27.7 \\
13 & $10: 01: 26.29$ & $-01: 34: 26.6$ & sdM5.0 & 115.8 & 82.8 \\
16 & $11: 58: 26.62$ & $+04: 47: 46.8$ & sdM9.5 & -123.6 & 3.8 \\
17 & $12: 02: 14.62$ & $+07: 31: 13.8$ & esdM7.0 & 284.6 & 222.3 \\
18 & $12: 15: 08.37$ & $+04: 02: 00.5$ & sdM7.0 & 51.8 & 76.7 \\
20 & $12: 36: 59.43$ & $-00: 21: 58.2$ & esdM5.5 & 219.5 & 68.9 \\
21 & $12: 42: 34.62$ & $+14: 33: 06.2$ & usdM5.0 & 97.6 & 7.4 \\
22 & $12: 44: 25.90$ & $+10: 24: 41.9$ & sdL0.5 & 248.0 & 236.4 \\
23 & $12: 46: 21.90$ & $+04: 43: 09.9$ & esdM5.0 & 127.9 & 100.6 \\
24 & $12: 56: 35.91$ & $-00: 19: 44.9$ & esdM6.0 & 185.5 & 126.7 \\
25 & $13: 17: 05.66$ & $+09: 10: 16.9$ & esdM7.0 & 83.4 & 12.9 \\
26 & $14: 18: 06.71$ & $+00: 00: 35.5$ & sdM6.5 & -115.8 & -161.0 \\
27 & $14: 54: 41.42$ & $+12: 35: 56.7$ & esdM5.5 & 128.6 & - \\
32 & $23: 33: 59.39$ & $+00: 49: 35.2$ & sdM6.0 & -163.9 & -192.9 \\
\hline
\end{tabular}

$d=$ 43.7-49.2 pc; Lépine et al. 2002; Schilbach et al. 2009), LSR J142504.81+710210.4 (sdM8; $J=14.828$ mag; $d=75.4-89.9$ pc; Lépine et al. 2003d; Burgasser et al. 2008; Schilbach et al. 2009), and SSSPM101307.34-135620.4 ( $\operatorname{sdM} 9.5 ; J=14.637 \mathrm{mag} ; d=45.0-54.6 \mathrm{pc}$; Scholz et al. 2004a; Schilbach et al. 2009). After applying the standard transformations using the $J$-band magnitudes of our new discoveries, we assigned mean distances between 88 and 628 pc (see Table 6), assuming that they are single. Typical error bars on the spectroscopic distances are 20-25\% taking into account the uncertainties on the trigonometric parallaxes of the templates. For the two L subdwarfs, we used the trigonometric parallax of the sdM9.5 template to place upper limits on their distances. Both objects very likely lie within $100 \mathrm{pc}$ unless they are binaries (Table 6).

In addition to the aforementioned subdwarfs, we were able to assign a spectroscopic distance for our esdM5 extreme subdwarf using LHS 515 as template (esdM5; $J=13.64$ mag; $d=42.6-64.5 \mathrm{pc}$ van Altena et al. 1995). Hence, we derive a spectroscopic distance of $257 \mathrm{pc}$ with a probable range of 214-323 pc for ULAS J124621.90+044309.9 (ID = 23; Table 6). For the other extreme subdwarfs we are unable to assign spectroscopic distances because no object with similar spectral types has a known trigonometric parallax in the literature. Instead, we used LHS 2096 (esdM5.5; $J=13.99 ; d=$ 56.10 pc; Lépine \& Shara 2005), LHS 2023 (esdM6; $J=14.91$; $d=73.9$ pc; Riaz et al. 2008), LSR J0822+1700 (esdM6.5; $J=15.517 ; d=106$ pc; Lépine et al. 2003c), and APMPM0559 (esdM7; $J=14.887 ; d=70 \mathrm{pc}$; Schweitzer et al. 1999) to infer tentative (mean) distances. We do not quote uncertainties for these sources because of the (already) very uncertain distances of the templates used. We do not provide a distance for the two ultra-subdwarfs in our sample because none has trigonometric parallaxes published in the literature. 
Table 6. Coordinates (in J2000), spectral types, and spectroscopic distance estimates (in pc) for our new subdwarfs.

\begin{tabular}{ccccc}
\hline \hline ID & $\begin{array}{c}\text { RA } \\
\text { hh:mm:ss.ss }\end{array}$ & $\begin{array}{c}\text { Dec } \\
{ }^{\prime}:^{\prime \prime}\end{array}$ & SpT & $\begin{array}{c}\text { Distance } \\
\text { pc }\end{array}$ \\
\hline 1 & $00: 45: 39.97$ & $+13: 50: 32.7$ & sdM7.5 & $354(334-376)$ \\
3 & $01: 33: 46.25$ & $+13: 28: 22.4$ & esdM6.5 & $124^{a}$ \\
4 & $01: 50: 34.33$ & $+14: 20: 02.4$ & sdM6.0 & $303(243-364)$ \\
5 & $02: 05: 33.75$ & $+12: 38: 24.1$ & sdM8.0 & $132(121-146)$ \\
6 & $03: 33: 50.84$ & $+00: 14: 06.1$ & sdL0.0 & $<97(88-107)$ \\
9 & $08: 43: 58.50$ & $+06: 00: 38.6$ & usdM5.5 & no template \\
10 & $08: 55: 00.12$ & $+00: 02: 04.1$ & sdM6.0 & $524(419-628)$ \\
13 & $10: 01: 26.29$ & $-01: 34: 26.6$ & sdM5.0 & $464(438-494)$ \\
16 & $11: 58: 26.62$ & $+04: 47: 46.8$ & sdM9.5 & $116(105-128)$ \\
17 & $12: 02: 14.62$ & $+07: 31: 13.8$ & esdM7.0 & $237^{a}$ \\
18 & $12: 15: 08.37$ & $+04: 02: 00.5$ & sdM7.0 & $281(274-288)$ \\
20 & $12: 36: 59.43$ & $-00: 21: 58.2$ & esdM5.5 & $171^{a}$ \\
21 & $12: 42: 34.62$ & $+14: 33: 06.2$ & usdM5.0 & no template \\
22 & $12: 44: 25.90$ & $+10: 24: 41.9$ & sdL0.5 & $<104(95-115)$ \\
23 & $12: 46: 21.90$ & $+04: 43: 09.9$ & esdM5.0 & $257(214-323)$ \\
24 & $12: 56: 35.91$ & $-00: 19: 44.9$ & esdM6.0 & $132^{a}$ \\
25 & $13: 17: 05.66$ & $+09: 10: 16.9$ & esdM7.0 & $214^{a}$ \\
25 & $13: 17: 05.66$ & $+09: 10: 16.9$ & esdM6.5 & $242^{a}$ \\
26 & $14: 18: 06.71$ & $+00: 00: 35.5$ & sdM6.5 & $464(424-517)$ \\
27 & $14: 54: 41.42$ & $+12: 35: 56.7$ & esdM5.5 & $150^{a}$ \\
32 & $23: 33: 59.39$ & $+00: 49: 35.2$ & sdM6.0 & $399(319-478)$ \\
\hline
\end{tabular}

Notes. ${ }^{(a)}$ The range in distances is not listed because the templates used do not have trigonometric parallaxes.

\subsection{Mid-infrared photometry}

We cross-matched our list of new ultracool subdwarfs with the Wide-field Infrared Survey Explorer (WISE; Wright et al. 2010) data release which took place in April 2011. We found two subdwarfs with mid-infrared photometry using a matching radius of 6.5 arcsec, the spatial resolution of WISE. However, we ensured that no other source was detected in WISE because of the large proper motions of subdwarfs. The WISE photometry of these two subdwarfs at 3.4 (W1) and 4.6 (W2) microns is reported in Table 7 and plotted in Fig. 10; these two sources are undetected at longer wavelengths.

We compared the infrared colours of our new ultracool subdwarfs to the sample of Kirkpatrick et al. (2011) drawn from the DwarfArchives.org webpage ${ }^{7}$ to identify any trend that may help future searches for metal-poor brown dwarfs. For ULAS J015034.33+142002.4 (ID = 4; sdM6), we find colours similar to normal M6 dwarfs in $H-\mathrm{W} 2=1.07, J-\mathrm{W} 2=0.76$, and $K-\mathrm{W} 1$ although on the red side of the distribution. Other colours, including $J-\mathrm{W} 1=0.76, \mathrm{~W} 1-\mathrm{W} 2=0.79, K-\mathrm{W} 2=$ 0.92 mag differ from those of normal M6 dwarfs, which span the following ranges: $1.0-1.4,0.2-0.4$, and $0.3-0.6$ mag, resulting in a deviation of $\sim 1 \sigma$ (Fig. 10; Table 7). The other subdwarf in our sample, ULAS145441.42+123556.7 (ID = 27; sdM5.5) exhibits similar colours as $\mathrm{M}$ dwarfs in all combinations of colours at odds with ULAS J015034.33+142002.4 (Fig. 10). We repeated the process with more known subdwarfs and did not spot any obvious trend with decreasing metallicity, suggesting that ULAS J015034.33+142002.4 may be peculiar, or a multiple source, or more likely the WISE photometry has underestimated uncertainties.

\footnotetext{
${ }^{7}$ http://spider.ipac.caltech.edu/staff/davy/ARCHIVE/ index.shtml
}

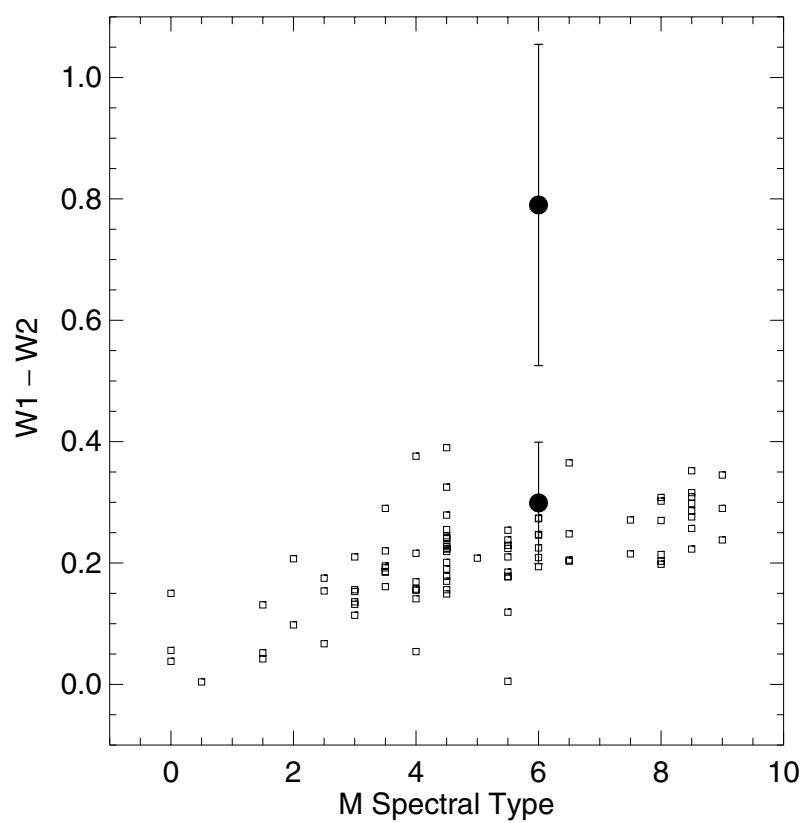

Fig. 10. WISE colours as a function of spectral type for known $\mathrm{M}$ dwarfs (open squares) from the sample published by Kirkpatrick et al. (2011). Our two subdwarf candidates are highlighted with large filled circles.

We also checked the Spitzer archive to extract additional information on the mid-infrared properties of some of our candidates but none of them was found in the Spitzer public database.

\subsection{Search for wide companions}

In this section we looked for wide companions brighter than each of our subdwarfs within a radius of 10 arcmin. The idea is to find potential primaries with distances, metallicities, and (possibly) ages (often referred to as benchmark objects; Pinfield et al. 2006) determined with a higher precision than our new subdwarfs. We used large-scale surveys with accurate proper motions: the USNO-B1 (Monet et al. 2003), the UCAC3 (Zacharias et al. 2010), and the PPMXL (Roeser et al. 2010) catalogues. We selected only bright sources with $I_{\text {USNO }} \leq 18$ mag and proper motions in right ascension and declination within $30 \%$ of the measured motion of our targets derived from the UKIDSS LAS DR5 vs. SDSS DR7 cross-match.

We found one potential wide companion to ULAS $\mathrm{J} 233359.39+004935.2$ (ID = 32) in USNO-B1 and PPMXL at a distance of about 5 arcmin on the sky. This potential companion, USNO J233350.27+005342.9, has a proper motion in right ascension and declination of $(149,-75)$ and $(154,-78)$ mas/yr in PPXML and USNO-B1, respectively, in agreement with the values of $(136,-71)$ mas/yr quoted by Sloan. The differences in the proper motions in right ascension and in declination between our subdwarf and its potential companion are about $23-27 \%$ and $10-15 \%$, respectively. No spectrum is available in the SDSS spectroscopic database. The SDSS $i$ of this object is $19.118 \pm 0.021 \mathrm{mag}$, roughly $1 \mathrm{mag}$ brighter than our spectroscopic subdwarf. Its optical colours $(g-r=1.785, r-i=1.429$, $r-z=2.158 \mathrm{mag})$ and reduced proper motion $\left(H_{r}=21.64 \mathrm{mag}\right)$ satisfy our original selection criteria and suggests that this potential wide companion may also be metal-poor. However, its $J-K$ infrared are redder than our original cut of 0.7 mag, implying that this object falls out of our sample. At the spectroscopic distance of ULAS J233359.39+004935.2 (ID = 32) estimated 
Table 7. Coordinates (in J2000), spectral type, $J$-band magnitude, and mid-infrared photometry with the associated error bars (3.4 and $4.6 \mu \mathrm{m}$ ) for the two ultracool subdwarfs covered by the WISE mission.

\begin{tabular}{lcccccc}
\hline \hline ID & $\begin{array}{c}\text { RA } \\
\text { hh:mm:ss.ss }\end{array}$ & $\begin{array}{c}\text { Dec } \\
:^{\prime}::^{\prime \prime}\end{array}$ & SpType & $\begin{array}{c}J \\
\mathrm{mag}\end{array}$ & $\begin{array}{c}3.4 \mu \mathrm{m}(\mathrm{SNR}) \\
\operatorname{mag} \pm \operatorname{err}()\end{array}$ & $\begin{array}{c}4.6 \mu \mathrm{m}(\text { SNR }) \\
\operatorname{mag} \pm \operatorname{err}()\end{array}$ \\
\hline 4 & $01: 50: 34.33$ & $+14: 20: 02.4$ & sdM6.0 & 17.424 & $16.667 \pm 0.131(8.3)$ & $15.877 \pm 0.230(4.7)$ \\
25 & $14: 54: 41.42$ & $+12: 35: 56.7$ & sdM5.5 & 16.121 & $15.136 \pm 0.044(24.4)$ & $14.837 \pm 0.090(12.1)$ \\
\hline
\end{tabular}

Notes. The signal-to-noise ratio of the photometry quoted by the WISE catalogue is indicated in brackets. The photometry in the WISE 12 and $22 \mu \mathrm{m}$ bands is not included because the signal-to-noise ratios are less than 3 .

to $400 \mathrm{pc}$ (Table 6), the projected physical separation of the system would be much larger, about $120000 \mathrm{au}$. Hence, we cannot claim that the two objects are gravitationally bound, but they might have formed in the same cluster or might belong to the same moving group.

\subsection{Notes on individual objects}

In this section we give additional details on a few specific candidates identified in our search for ultracool subdwarfs.

\subsubsection{Spectra in the SDSS DR7 spectroscopic database}

Some photometric candidates in our sample have spectra publicly available in the SDSS DR7 spectroscopic database (Table 3). As noted in the caption of Table 1, all of them were included in the sample of West et al. (2008) and were classified as early-M dwarfs using the Hammer classification (Covey et al. 2007). However, the Sloan spectra clearly look like subdwarfs with the strong $\mathrm{CaH}$ and $\mathrm{TiO}$ bands typical of low-metallicity M dwarfs (Figs. 4,5). We should mention that LP 468-277 $(01: 33: 46.25+13: 28: 22.4)$ was included in the catalogue of Northern stars of Lépine \& Shara (2005), but no spectral type was derived. Finally, we recovered SDSS J020533.75+123824.1 (ID = 5) classified as sdM7.5 by Lépine \& Scholz (2008) and re-classified as sdM8 in this paper based on the spectral type provided by the SDSS DR7 spectroscopic database.

\subsubsection{ULAS J145441.45+123557.6 (ID = 27)}

This source is part of the catalogue of Northern stars of Lépine \& Shara (2005), but no spectral type was derived. Our proper motion derived from the LAS and SDSS DR7 epochs (0.31 arcsec/yr) agrees well with the value of $0.321 \mathrm{arcsec} / \mathrm{yr}$ published by Lépine \& Shara (2005). We do not have spectroscopic followup for this source yet.

\subsubsection{ULAS J233359.39+004935.2 (ID = 32)}

This candidate, observed with VLT FORS2, is classified as a sdM6 subdwarf. Its proper motion in right ascension and declination is $(93.9,23.0)$ and $(81.9,16.6) \mathrm{mas} / \mathrm{yr}$ from the 2MASS/UKIDSS and SDSS/UKIDSS cross-match. We discuss a possible wide companion five arcmin away in Sect. 5.5.

This subdwarf is located at $\sim 42$ arcsec from 2MASS J233358.40+005011.9, a L0 dwarf reported by Zhang et al. (2010) with a proper motion of $(139.7,29.5) \mathrm{mas} / \mathrm{yr}$ reported in the PPMXL catalogue (Roeser et al. 2010). The proper motion values in right ascension differ by $50-70 \%$, suggesting that the two objects are not physically associated. Nonetheless, we investigated the SDSS DR7 spectrum of this possible wide companion in more details and compared it to known M9 and M9.5 dwarfs,

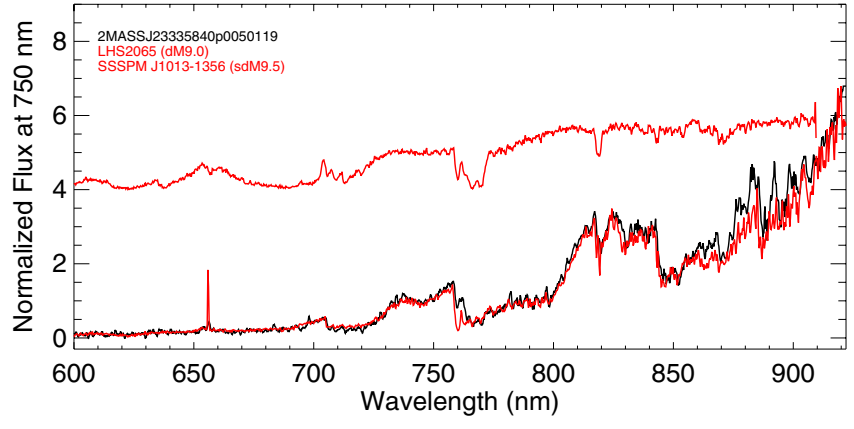

Fig. 11. Comparison of 2MASS J233358.40+005011.9 classified as L0 by Zhang et al. (2010) with a known solar metallicity, LHS 2065, classified as M9 by Kirkpatrick et al. (1991) and a sdM9.5 subdwarf (Scholz et al. 2004a).

young L-dwarf templates (Cruz et al. 2009), a sdM9.5 subdwarf (Scholz et al. 2004a), and our two possible L subdwarfs (Fig. 11). The best fit is obtained for a field M9 dwarf of solar metallicity, LHS 2065 (Kirkpatrick et al. 1991) downloaded from Kelle Cruz's webpage (Fig. 11) ${ }^{8}$, earlier by one subclass compared to the classification by Zhang et al. (2010).

Overall conclusion: these two sources which are separated by about 42 arcsec, are unlikely to be physically associated. Note that we also discussed in the previous section (Sect. 5.5) that this object may be part of a wide binary system or an old moving group.

\subsubsection{ULAS J033350.84+001406.1 (ID =6)}

This bright source $(J=16.1 \mathrm{mag})$ has a large proper motion of $0.78 \mathrm{arcsec} / \mathrm{yr}$. Its VLT FORS2 spectrum appears to be slightly redder than ULAS J115826.62+044746.8 (ID = 16), which is well reproduced by the IRTF/SpeX spectrum of SSSPM 1013-1356 (sdM9.5; Scholz et al. 2004a; Burgasser 2004). Hence, we propose this object as a new sdL0 template for future searches because it is the first of its class known to date.

\subsubsection{ULAS J124425.90+102441.9 (ID = 22)}

This bright $(J=16.26 \mathrm{mag}$ ) candidate is the coolest object in our sample, cooler than ULAS J033350.84+001406.1 (ID = 6; see previous section). Hence we propose it as a sdL0.5 template for future works on subdwarfs because it is the first of its class at the time of writing. Its proper motion also exceeds the $0.5 \mathrm{arcsec} / \mathrm{yr}$ threshold of high proper motion sources, with a total motion of $0.67 \mathrm{arcsec} / \mathrm{yr}$. We note that the two new targets classified as L subdwarfs occupy the lower right of the reduced proper motion diagram (Fig. 2), suggesting a steep reddening in the colours of subdwarfs in the $\mathrm{M} / \mathrm{L}$ transition.

\footnotetext{
${ }^{8}$ http://kellecruz.com/M_standards/
} 


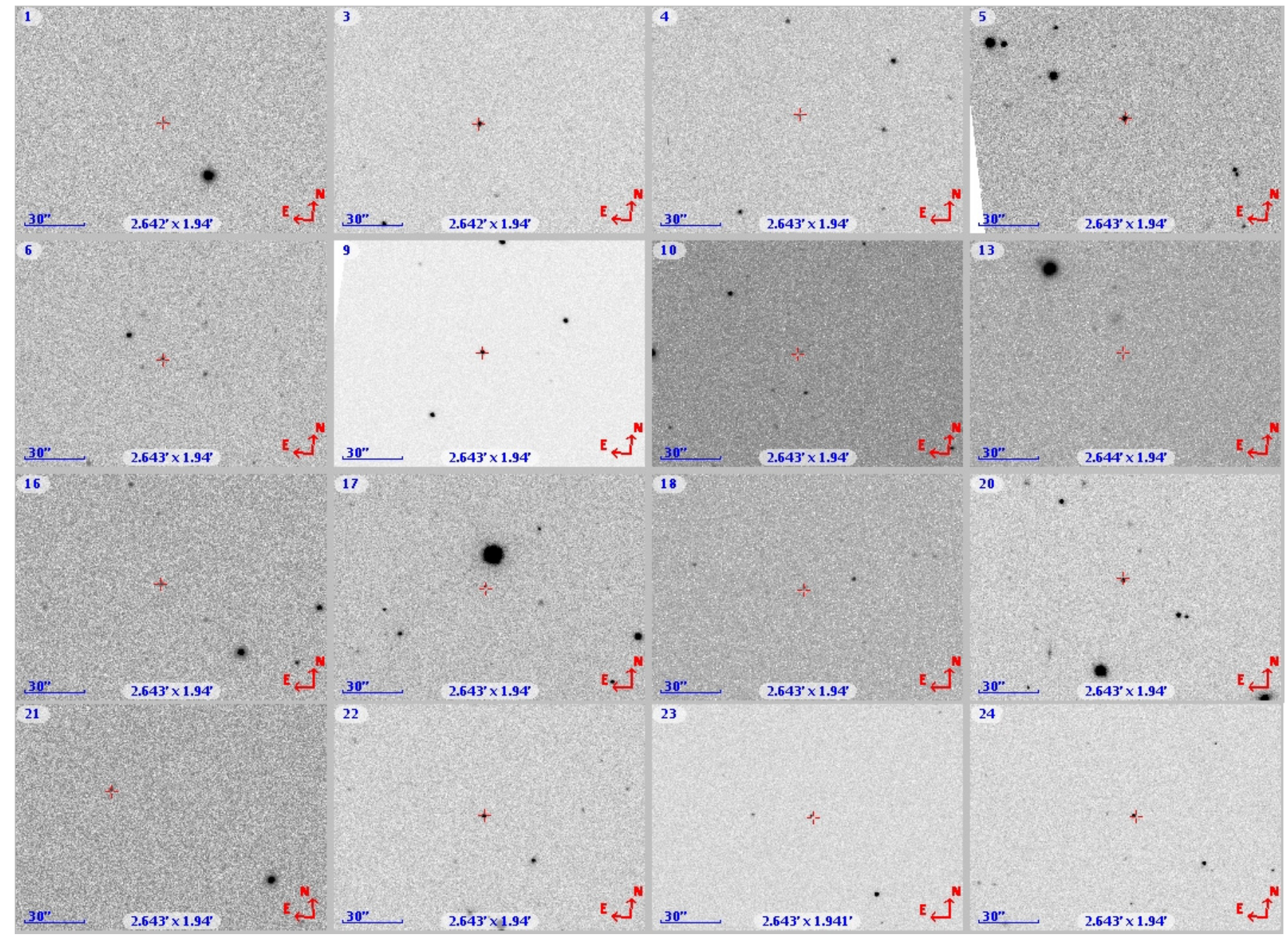

Fig. A.1. Finding charts for 16 confirmed subdwarfs. Images are in the Sloan $z$-band and approximately $2.64 \times 1.94$ arcmin aside with North up and East left. The ID number of the object is indicated at the top left of the image and the scale at the bottom left.

\subsection{Surface density of subdwarfs}

In this section, we provide a tentative estimate of the surface density of ultracool subdwarfs, i.e. metal-poor dwarfs with spectral types later than M5. We found a total of 18 M5-M9.5 subdwarfs (and two additional subdwarfs classified as L0-L0.5) over 1343 square degrees common to UKIDSS LAS DR5 and SDSS DR7 and imaged in the $Y J H K$ filters. We should potentially add one source that has no spectroscopy yet. We derive a density of $0.015-0.016 \geq$ M5 subdwarfs per square degree.

West et al. (2011) identified 70841 spectroscopic M0M9 dwarfs in the 8200 square degrees covered by the SDSS spectroscopic database. However, the SDSS spectroscopic follow-up of the imaging survey is incomplete. For this reason, we considered the photometric sample from Bochanski et al. (2010, their Fig. 4) and focused on sources with $i$ brighter than $22 \mathrm{mag}, r-z \geq 2.5, i-z \geq 0.2$, and $r-i \geq 0.3 \mathrm{mag}$, corresponding to $\geq \mathrm{M} 5$ dwarfs and later. We counted a total number of 653625 photometric $\geq$ M5 dwarfs in 8000 square degrees surveyed by SDSS DR $5^{9}$, implying a density of $\sim 82 \geq$ M5 dwarfs per square degree. We sent a query with the aforementioned criteria to the WFCAM Science Archive (Hambly et al. 2008) to see how many M5 (and later) dwarfs in UKIDSS LAS DR5 and SDSS DR7 we could recover to match those numbers with our

\footnotetext{
9 Numbers kindly provided by John Bochanski and Andrew West.
}

search criteria defined in Sect. 2. We imposed a detection in $Y J H$ but not in $K$ and requested good-quality point sources in addition to the constraints on the optical colours. The query returned 113393 sources in 1343 square degrees. If we limit the sample to dwarfs with $z-J$ greater than 1.4 (West et al. 2011), the query returns 106746 sources, implying a number of M5 dwarfs (and later) about 79.5-84.4 per square degree, which is highly consistent with the numbers derived from the Sloan sample alone. This density is $\sim 5000-5700$ times higher than the number of ultracool subdwarfs found in our photometric and proper motion search, which is broadly consistent with the $0.2 \%$ contribution from metal-poor stars quoted by Digby et al. (2003) and the upper limit derived from the SDSS M dwarf sample (Covey et al. 2008). We should mention that according to the evolutionary models (Baraffe et al. 1997, 1998), the masses of $\geq$ M5 dwarfs and subdwarfs are similar at ages of Gyr, but lower metallicity M dwarfs have high effective temperatures. Finally, we point out that we found two ultra-subdwarf and seven extreme subdwarfs for 11 subdwarfs, suggesting a fairly quick decrease in the numbers of subdwarfs as a function of metallicity.

\section{Summary}

We have presented the outcome of a dedicated photometric and proper motion search aimed at finding new ultracool subdwarfs 


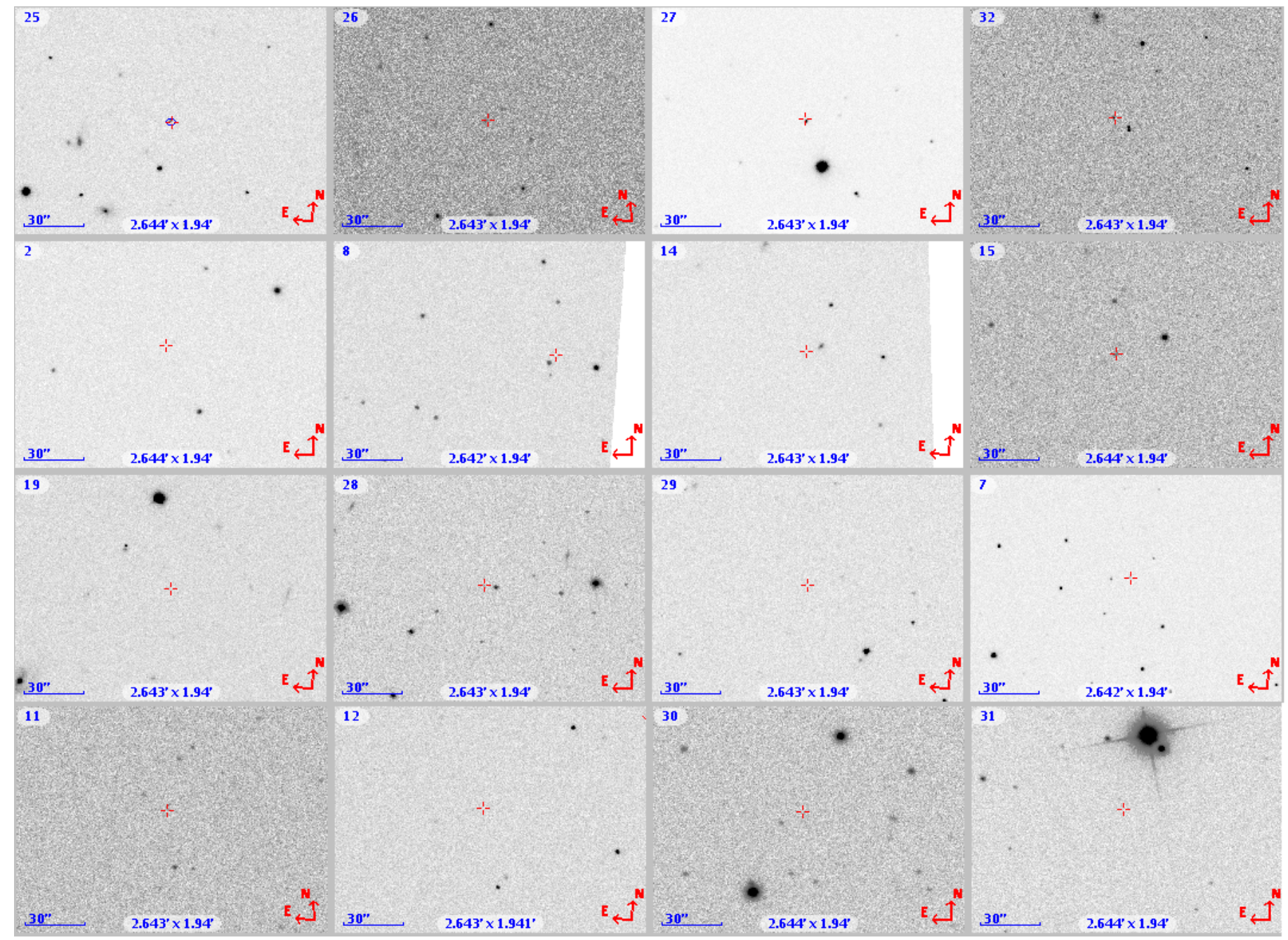

Fig. A.2. The other 16 candidates identified in this work: the remaining four subdwarfs confirmed spectroscopically are shown at the top, the seven solar-metallicity M dwarfs below followed by the five candidates without optical spectra. Images are in the Sloan $z$-band and approximately $2.64 \times 1.94$ arcmin aside with North up and East left. The ID number of the object is indicated at the top left of the image and the scale at the bottom left.

in public databases. We identified 32 ultracool subdwarf candidates, 20 of them being confirmed as metal-poor late-M and early-L dwarfs by low-resolution optical spectroscopy. We discovered two new early-L subdwarfs, which we propose as spectroscopic templates for future searches because these are the first of their subclass. We measured radial velocities for most of the new subdwarfs with the cross-match technique. We estimated their spectroscopic distances when templates of similar spectral types with trigonometric parallaxes were available. We uncovered seven old $\mathrm{M}$ dwarfs that contaminate our sample, whose ages are estimated to $>5-8$ Gyr owing to the lack of $\mathrm{H} \alpha$ in emission. Of the 32 candidates, five do not have optical spectroscopy. Only one of these five remains a good subdwarf candidate, the others were rejected. We found a contamination of about $30 \%$ by solar-metallicity $\mathrm{M}$ dwarfs in our photometric and proper motion search, mainly due to large errors on the Sloan positions, which led to spurious proper motions that affected the determination of the reduced proper motion. We were able to reduce this level of contamination by a factor 2 to 3 after revision of the proper motion measurements. We also presented mid-infrared data from WISE for two subdwarfs as well as a search for bright and wide common proper motions, which led to an extremely wide pair that is very likely not gravitationally bound.
We intend to expand our search for subdwarfs with upcoming data releases from UKIDSS to increase the census of lowmetallicity dwarfs. Moreover, we plan to update our colour criteria to optimise future searches and discover even cooler ultracool subdwarfs. The main overall scientific goal of this large project is to update and extend the current low-metallicity classification into the $\mathrm{L}$ dwarf (and later $\mathrm{T}$ dwarf) regime.

Acknowledgements. N.L. was funded by the Ramón y Cajal fellowship number 08-303-01-02 and the national program AYA2010-19136 funded by the Spanish ministry of science and innovation. N.L. was partly funded by the RoPACS (Rocky Planets Around Cool Stars) Marie Curie Initial Training Network. This research has made use of the Spanish Virtual Observatory supported from the Spanish MICINN through grant AYA2008-02156. Additional support was provided by the CONSOLIDER-INGENIO GTC project. We thank John Bochanski and Andrew West, who kindly provided the numbers of M dwarfs found in Sloan. We would like to thank the anonymous referee for her/his valuable comment on proper motions. The data presented here were obtained [in part] with ALFOSC, which is provided by the Instituto de Astrofísica de Andalucia (IAA) under a joint agreement with the University of Copenhagen and the NBIfAFG of the Astronomical Observatory of Copenhagen. This article is partly based on observations obtained in service mode with the Nordic Optical Telescope, operated on the island of La Palma jointly by Denmark, Finland, Iceland, Norway, and Sweden, in the Spanish Observatorio del Roque de los Muchachos of the Instituto de Astrofísica de Canarias. We would like to thank Jorge Garcia Rojas and the entire Support Astronomer Group for taking data in service mode for us. This work made use of data taken within the framework of the UKIRT Infrared 
Deep Sky Survey (UKIDSS). The United Kingdom Infrared Telescope is operated by the Joint Astronomy Centre on behalf of the UK Science Technology and Facility Council. This research has made use of the Simbad and Vizier databases, operated at the Centre de Données Astronomiques de Strasbourg (CDS), and of NASA's Astrophysics Data System Bibliographic Services (ADS). This publication makes use of data products from the Two Micron All Sky Survey (2MASS), which is a joint project of the University of Massachusetts and the Infrared Processing and Analysis Center/California Institute of Technology, funded by the National Aeronautics and Space Administration and the National Science Foundation. Funding for the SDSS and SDSS-II has been provided by the Alfred P. Sloan Foundation, the Participating Institutions, the National Science Foundation, the US Department of Energy, the National Aeronautics and Space Administration, the Japanese Monbukagakusho, the Max Planck Society, and the Higher Education Funding Council for England. The SDSS Web Site is http://www.sdss.org/. The SDSS is managed by the Astrophysical Research Consortium for the Participating Institutions. The Participating Institutions are the American Museum of Natural History, Astrophysical Institute Potsdam, University of Basel, University of Cambridge, Case Western Reserve University, University of Chicago, Drexel University, Fermilab, the Institute for Advanced Study, the Japan Participation Group, Johns Hopkins University, the Joint Institute for Nuclear Astrophysics, the Kavli Institute for Particle Astrophysics and Cosmology, the Korean Scientist Group, the Chinese Academy of Sciences (LAMOST), Los Alamos National Laboratory, the Max-PlanckInstitute for Astronomy (MPIA), the Max-Planck-Institute for Astrophysics (MPA), New Mexico State University, Ohio State University, University of Pittsburgh, University of Portsmouth, Princeton University, the United States Naval Observatory, and the University of Washington. The DENIS project has been partly funded by the SCIENCE and the HCM plans of the European Commission under grants CT920791 and CT940627. It is supported by INSU, MEN and CNRS in France, by the State of Baden-Württemberg in Germany, by DGICYT in Spain, by CNR in Italy, by FFwFBWF in Austria, by FAPESP in Brazil, by OTKA grants F-4239 and F-013990 in Hungary, and by the ESO C\&EE grant A-04-046. Jean Claude Renault from IAP was the Project manager. Observations were carried out thanks to the contribution of numerous students and young scientists from all involved institutes, under the supervision of $\mathrm{P}$. Fouqué, survey astronomer resident in Chile. This research has made use of data obtained from the SuperCOSMOS Science Archive, prepared and hosted by the Wide Field Astronomy Unit, Institute for Astronomy, University of Edinburgh, which is funded by the UK Particle Physics and Astronomy Research Council. This publication makes use of data products from the Wide-field Infrared Survey Explorer, which is a joint project of the University of California, Los Angeles, and the Jet Propulsion Laboratory/California Institute of Technology, funded by the National Aeronautics and Space Administration. This research has benefitted from the M, L, and T dwarf compendium housed at DwarfArchives.org and maintained by Chris Gelino, Davy Kirkpatrick, and Adam Burgasser. This research has benefitted from the SpeX Prism Spectral Libraries, maintained by Adam Burgasser at http://www. browndwarfs.org/spexprism. This research has also benefitted from the $\mathrm{M}$ dwarf standard spectra made available by Kelle Cruz at www. astro.caltech.edu/ kelle/M_standards/.

\section{References}

Abazajian, K. N., Adelman-McCarthy, J. K., Agüeros, M. A., et al. 2009, ApJS, 182,543

Adelman-McCarthy, J. K., et al. 2009, VizieR Online Data Catalog, 2294, 0 Albert, L., Artigau, É., Delorme, P., et al. 2011, AJ, 141, 203

Appenzeller, I., Fricke, K., Fürtig, W., et al. 1998, The Messenger, 94, 1 Baraffe, I., Chabrier, G., Allard, F., \& Hauschildt, P. H. 1997, A\&A, 327, 1054 Baraffe, I., Chabrier, G., Allard, F., \& Hauschildt, P. H. 1998, A\&A, 337, 403 Bochanski, J. J., Hawley, S. L., Covey, K. R., et al. 2010, AJ, 139, 2679 Bonnarel, F., Fernique, P., Bienaymé, O., et al. 2000, A\&AS, 143, 33 Bowler, B. P., Liu, M. C., \& Dupuy, T. J. 2010, ApJ, 710, 45 Burgasser, A. J. 2004, ApJ, 614, L73

Burgasser, A. J., Kirkpatrick, J. D., Brown, M. E., et al. 2002, ApJ, 564, 421 Burgasser, A. J., Kirkpatrick, J. D., Burrows, A., et al. 2003, ApJ, 592, 1186 Burgasser, A. J., Cruz, K. L., \& Kirkpatrick, J. D. 2007, ApJ, 657, 494 Burgasser, A. J., Vrba, F. J., Lépine, S., et al. 2008, ApJ, 672, 1159 Burgasser, A. J., Witte, S., Helling, C., et al. 2009, ApJ, 697, 148 Burningham, B., Pinfield, D. J., Lucas, P. W., et al. 2010, MNRAS, 406, 1885 Covey, K. R., Ivezić, Ž., Schlegel, D., et al. 2007, AJ, 134, 2398

Covey, K. R., Hawley, S. L., Bochanski, J. J., et al. 2008, AJ, 136, 1778 Cruz, K. L., Kirkpatrick, J. D., \& Burgasser, A. J. 2009, AJ, 137, 3345 Cushing, M. C., Looper, D., Burgasser, A. J., et al. 2009, ApJ, 696, 986 Cushing, M. C., Kirkpatrick, J. D., Gelino, C. R., et al. 2011, ApJ, 743, 50 Cutri, R. M., Skrutskie, M. F., van Dyk, S., et al. 2003, 2MASS All Sky Catalog of point sources, 2246
Delfosse, X., Tinney, C. G., Forveille, T., et al. 1997, A\&A, 327, L25 Delfosse, X., Tinney, C. G., Forveille, T., et al. 1999, A\&AS, 135, 41 Delorme, P., Delfosse, X., Albert, L., et al. 2008, A\&A, 482, 961 DENIS Consortium 2005, VizieR Online Data Catalog, 1, 2002

Digby, A. P., Hambly, N. C., Cooke, J. A., Reid, I. N., \& Cannon, R. D. 2003, MNRAS, 344, 583

Evans, N. W. 1992, MNRAS, 258, 587

Fan, X., Knapp, G. R., Strauss, M. A., et al. 2000, AJ, 119, 928

Geballe, T. R., Knapp, G. R., Leggett, S. K., et al. 2002, ApJ, 564, 466

Gizis, J. E. 1997, AJ, 113, 806

Gizis, J. E., \& Reid, I. N. 1997, PASP, 109, 849

Hambly, N. C., Davenhall, A. C., Irwin, M. J., \& MacGillivray, H. T. 2001a, MNRAS, 326, 1315

Hambly, N. C., Irwin, M. J., \& MacGillivray, H. T. 2001b, MNRAS, 326, 1295

Hambly, N. C., MacGillivray, H. T., Read, M. A., et al. 2001c, MNRAS, 326, 1279

Hambly, N. C., Collins, R. S., Cross, N. J. G., et al. 2008, MNRAS, 384, 637

Hewett, P. C., Warren, S. J., Leggett, S. K., \& Hodgkin, S. T. 2006, MNRAS, 367,454

Jao, W.-C., Henry, T. J., Beaulieu, T. D., \& Subasavage, J. P. 2008, AJ, 136, 840 Jones, E. M. 1972, ApJ, 177, 245

Kirkpatrick, J. D., Henry, T. J., \& McCarthy, D. W. 1991, ApJS, 77, 417

Kirkpatrick, J. D., Reid, I. N., Liebert, J., et al. 2000, AJ, 120, 447

Kirkpatrick, J. D., Looper, D. L., Burgasser, A. J., et al. 2010, ApJS, 190, 100

Kirkpatrick, J. D., Cushing, M. C., Gelino, C. R., et al. 2011, ApJS, 197, 19

Latham, D. W., Stefanik, R. P., Torres, G., et al. 2002, AJ, 124, 1144

Lawrence, A., Warren, S. J., Almaini, O., et al. 2007, MNRAS, 379, 1599

Leggett, S. K., Geballe, T. R., Fan, X., et al. 2000, ApJ, 536, L35

Lépine, S., \& Scholz, R.-D. 2008, ApJ, 681, L33

Lépine, S., \& Shara, M. M. 2005, AJ, 129, 1483

Lépine, S., Shara, M. M., \& Rich, R. M. 2002, AJ, 124, 1190

Lépine, S., Rich, R. M., \& Shara, M. M. 2003a, ApJ, 591, L49

Lépine, S., Rich, R. M., \& Shara, M. M. 2003b, AJ, 125, 1598

Lépine, S., Shara, M. M., \& Rich, R. M. 2003c, AJ, 126, 921

Lépine, S., Shara, M. M., \& Rich, R. M. 2003d, ApJ, 585, L69

Lépine, S., Rich, R. M., \& Shara, M. M. 2007, ApJ, 669, 1235

Lodieu, N., Scholz, R.-D., McCaughrean, M. J., et al. 2005, A\&A, 440, 1061

Lodieu, N., Pinfield, D. J., Leggett, S. K., et al. 2007, MNRAS, 379, 1423

Lodieu, N., Leggett, S. K., Bergeron, P., \& Nitta, A. 2009, ApJ, 692, 1506

Lodieu, N., Zapatero Osorio, M. R., Martín, E. L., Solano, E., \& Aberasturi, M. 2010, ApJ, 708, L107

Luyten, W. J. 1979, LHS catalogue. A catalogue of stars with proper motions exceeding $0^{\prime \prime} 5$ annually, 2nd edn. (Minneapolis: University of Minnesota)

Luyten, W. J. 1980, NLTT catalogue. 0 deg. to -10 deg. (Minneapolis: University of Minnesota), 3

Martín, E. L., Delfosse, X., Basri, G., et al. 1999, AJ, 118, 2466

Monet, D. G., Levine, S. E., Canzian, B., et al. 2003, AJ, 125, 984

Pinfield, D. J., Jones, H. R. A., Lucas, P. W., et al. 2006, MNRAS, 368, 1281

Pinfield, D. J., Burningham, B., Tamura, M., et al. 2008, MNRAS, 390, 304

Reid, I. N., \& Gizis, J. E. 2005, PASP, 117, 676

Reylé, C., Delorme, P., Willott, C. J., et al. 2010, A\&A, 522, A112

Riaz, B., Gizis, J. E., \& Samaddar, D. 2008, ApJ, 672, 1153

Roeser, S., Demleitner, M., \& Schilbach, E. 2010, AJ, 139, 2440

Ryan, S. G. 1989, AJ, 98, 1693

Salim, S., \& Gould, A. 2002, ApJ, 575, L83

Salim, S., \& Gould, A. 2003, ApJ, 582, 1011

Schilbach, E., Röser, S., \& Scholz, R. 2009, A\&A, 493, L27

Schmidt, S. J., West, A. A., Burgasser, A. J., Bochanski, J. J., \& Hawley, S. L. 2010a, AJ, 139, 1045

Schmidt, S. J., West, A. A., Hawley, S. L., \& Pineda, J. S. 2010b, AJ, 139, 1808

Scholz, R.-D., Irwin, M., Ibata, R., Jahreiß, H., \& Malkov, O. Y. 2000, A\&A, 353,958

Scholz, R., Lehmann, I., Matute, I., \& Zinnecker, H. 2004a, A\&A, 425, 519

Scholz, R.-D., Lodieu, N., \& McCaughrean, M. J. 2004b, A\&A, 428, L25

Schweitzer, A., Scholz, R.-D., Stauffer, J., Irwin, M., \& McCaughrean, M. J. 1999, A\&A, 350, L62

Sivarani, T., Lépine, S., Kembhavi, A. K., \& Gupchup, J. 2009, ApJ, 694, L140

Skrutskie, M. F., Cutri, R. M., Stiening, R., et al. 2006, AJ, 131, 1163

van Altena, W. F., Lee, J. T., \& Hoffleit, E. D. 1995, The general catalogue of trigonometric [stellar] parallaxes, ed. W. F. van Altena, J. T. Lee, \& E. D. Hoffleit

West, A. A., Hawley, S. L., Bochanski, J. J., et al. 2008, AJ, 135, 785

West, A. A., Morgan, D. P., Bochanski, J. J., et al. 2011, AJ, 141, 97

Woolf, V. M., Lépine, S., \& Wallerstein, G. 2009, PASP, 121, 117

Wright, E. L., Eisenhardt, P. R. M., Mainzer, A. K., et al. 2010, AJ, 140, 1868

Yong, D., \& Lambert, D. L. 2003, PASP, 115, 796

Zacharias, N., Finch, C., Girard, T., et al. 2010, AJ, 139, 2184

Zhang, Z. H., Pinfield, D. J., Day-Jones, A. C., et al. 2010, MNRAS, 404, 1817 